\title{
Scalability Study of Backhaul Capacity Sensitive Network Selection Scheme in LTE-WiFi HetNet
}

\author{
Alvin Ting, David Chieng, \\ Kae Hsiang Kwong \\ Wireless Communication, MIMOS \\ Berhad, K.L, Malaysia. \{kee.ting, \\ ht.chieng, kh.kwong\}@mimos.my
}

\author{
Ivan Andonovic \\ CIDCOM, Dept. EEE, University of \\ Strathclyde, Scotland, UK \\ i.andonovic@eee.strath.ac.uk
}

\author{
K. D. Wong \\ Daniel Wireless LLC \\ Palo Alto, CA, USA \\ dwong@danielwireless.com
}

\begin{abstract}
Wireless Heterogeneous Network (HetNet) with small cells presents a new backhauling challenge which differs from those of experienced by conventional macro-cells. In practice, the choice of backhaul technology for these small cells whether fiber, xDSL, point-to-point and point-to-multipoint wireless, or multi-hop/mesh networks, is often governed by availability and cost, and not by required capacity. Therefore, the resulting backhaul capacity of the small cells in HetNet is likely to be non-uniform due to the mixture of backhaul technologies adopted. In such an environment, a question then arises whether a network selection strategy that considers the small cells' backhaul capacity will improve the end users' usage experience. In this paper, a novel Dynamic Backhaul Capacity Sensitive (DyBaCS) network selection schemes (NSS) is proposed and compared with two commonly used network NSSs, namely WiFi First (WF) and Physical Data Rate (PDR) in an LTE-WiFi HetNet environment. The proposed scheme is evaluated in terms of average connection or user throughput ${ }^{1}$ and fairness among users. The effects of varying WiFi backhaul capacity (uniform and non-uniform distribution), WiFi-LTE coverage ratio, user density and WiFi access points (APs) density within the HetNet form the focus of this paper. Results show that the DyBaCS scheme generally provides superior fairness and user throughput performance across the range of backhaul capacity considered. Besides, DyBaCS is able to scale much better than WF and PDR across different user and WiFi densities.
\end{abstract}

Keywords - Hetrogeneous Network, Network Selection, LTE, WiFi, Traffic Offload, Backhaul Capacity

\section{Introduction}

The increasing pressure for mobile operators to offload data traffic from their $3 \mathrm{G}$, LTE or WiMAX networks to small cell networks [1] [2] indicates that future mobile broadband networks will largely be heterogeneous. This migration is further fueled by the availability of multi-RAT (Radio Access Technology) which allows user devices to connect to different wireless networks such as 3G, LTE, WiMAX, WiFi either one at a time or simultaneously. Deployment of small cells such as WiFi raises new backhaul challenges for operators. There are only two broad choices as far as backhaul is concerned,

${ }^{1}$ Capacity and throughput are used interchangeably throughout article with the detail definition in section 2.1 wireline and wireless backhaul. Due to intensive engineering work required, high cost and regulatory barriers, fixed line solutions such as fiber, cable, copper or xDSL are not ubiquitously available. Further, a relatively large number of $\mathrm{WiFi}$ hotspots may prove too costly for operators to backhaul with wired options. In such situations, the operator may instead increasingly rely on point-to-point and point-to-multipoint wireless solutions. However in most scenarios, a mixture of backhaul technologies is expected, as operators will adopt the most suitable backhaul solution for small cells considering the cost and availability [3] (Figure 1). With the maturity of multihop mesh networks, it may also serve as a potential candidate [4] [5].

Currently, WiFi APs are typically backhauled through different types of technologies which offer throughput capacity ranging from several mega bit per second (Mbps) to tens of Mbps [4]. Backhauling APs using different technologies within the HetNet leads to non-uniform AP capacity distribution. The most widely deployed IEEE802.11n WiFi technology which provisions a peak physical data rate of $600 \mathrm{Mbps}$. Due to that most existing fixed backhaul services are not able to offer sufficient capacity for these WiFi APs to realize their full potential. Therefore consideration of WiFi backhaul capacity is inevitable during traffic offload decision making.

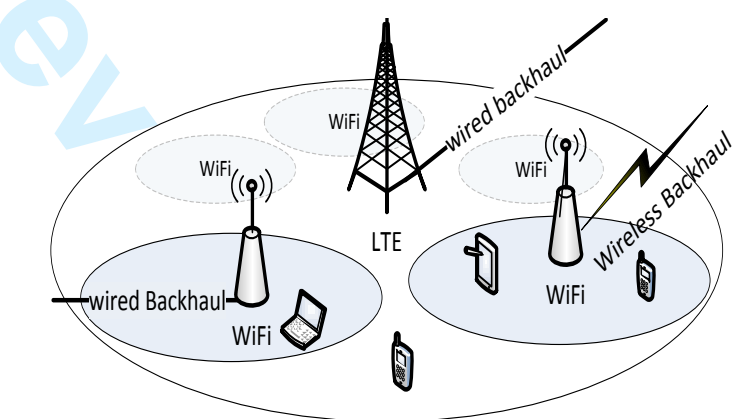

Figure 1: LTE-WiFi Heterogeneous Network with different backhaul options.

\subsection{Related Work}

To reduce data traffic pressure over mobile networks, alternative networks such as WiFi are preferred by mobile operators whenever possible [6]. As report in [7], most WiFi-enabled smartphones are configured with WiFi First network selection scheme by default to give WiFi higher priority over the cellular interface for data transmissions. Network selection strategies for WiMAX-WiFi network which 
is predominantly driven by data rate are reported in [8] and [9]. This kind of network selection strategies lead to poor user experiences [10] and causes unbalanced load distribution amongst access networks [11].

Several studies [12-14] have proposed the use of Network Selection Schemes (NSSs) to optimize network performance. The work by [12] considers a globally optimal user-network association scheme in a WLAN-UMTS hybrid cell. In [13], a heuristic greedy search algorithm that maximizes total user throughput in a heterogeneous wireless access network comprising WiFi APs and 3G BSs is proposed. Both these studies adopt a simplified WLAN model by assuming only one transmission rate. Poisson point process (PPP) theory and stochastic geometry method are used in [14] to model traffic offloading in a multi RAT heterogeneous wireless network. The authors propose a method to determine the optimum percentage of the traffic that needs be offloaded in order to maximize network coverage while meeting user requirements. In that work however, no network selection algorithm is proposed.

Fairness performance is considered in [15-18] where [15] focuses on ensuring max-min fairness for multicast in Orthogonal Frequency Division Multiple Access (OFDMA)based wireless heterogeneous networks. A proportional user rate based radio resource management strategy is investigated in [16] on LTE-WiFi HetNet, where a suboptimal network selection algorithm is introduced to improve the minimum normalized user rate and fairness. Bejerano et al. [17] proposes changes to the transmission power of AP beacon messages in order to minimize the load in congested APs and produces an optimal max-min load balancing strategy. A load balancing scheme for overlapping wireless LAN cells is reported in [18]. However, the work aims to balance the throughput of APs and not the fairness amongst users.

NSSs that consider QoS are reported in [6] [22] [23]. Network selection based on multiple parameters such as cost, bandwidth and QoS parameters including packet loss, jitter and delay is reported in [22]. However, the study focuses only on general heterogeneous networking and no specific type of technology is simulated. Similarly, the work in [6] and [23] investigate access network selection for optimal service delivery and QoS to users respectively. However the overall HetNet performance and fairness is not considered.

Load balancing in Cellular and WLAN HetNet is reported in [19] [20] and [21]. In [19], a joint access-control strategy is designed for sharing of the radio resource and load balancing between CDMA Cellular Network and WLANs by considering user preferences. Bandwidth allocation is optimized by maximizing the aggregated social welfare of the WLANs under interference-constraint environment. However, the method used to balance the load amongst mobile nodes is not disclosed. The work in [20] looks into the trade-off between the amount of traffic being offloaded and users' satisfaction. An incentive framework is proposed to motivate users to use delay tolerance WiFi networks for traffic offloading. Yang et al. in [21] proposes a load balancing scheme that aims to balance the network load between the LTE network and WiFi hotspots considering access pattern of UEs.
Bejerano et al. [10] considers backhaul capacity during AP selection, while [24] proposes a backhaul-aware base station (BS) selection algorithm. Although backhaul-aware network selection scheme have been explored in these works, their scope is limited to homogeneous wireless networks.

In this paper, a Dynamic Backhaul Capacity Sensitive (DyBaCS) NSS is proposed for LTE-WiFi HetNet. This scheme not only considers access link throughput but also available backhaul capacity in order to preserve fairness amongst users. The proposed scheme is compared with two commonly used NSSs namely WiFi First (WF) and Physical Data Rate (PDR). This paper investigates fairness and average user throughput performances across WiFi backhaul capacity ranging from $1 \mathrm{Mbps}$ to $25 \mathrm{Mbps}$.

The rest of the paper is organized as follows. Section 2 describes the proposed DyBaCS network selection algorithm as well as WF and PDR schemes. Section 3 details the simulation approach, simulation parameters and assumptions made. Results are discussed in Section 4 followed by conclusions in Section 5.

\section{Existing and Proposed Network Selection Schemes}

This section describes the simulation approach simulation parameters and assumptions used in the study. The notations used are listed in TABLE I.

\begin{tabular}{|c|c|}
\hline Notation & Description \\
\hline$N_{\text {cluster }}$ & number of clusters for user distribution \\
\hline$\sigma_{x}, \sigma_{y}$ & width and height of user distribution clusters \\
\hline$N_{\text {user }}$ & number of users in a cluster \\
\hline SINR & signal to interference and noise ratio \\
\hline SNR & signal to noise ratio \\
\hline$p_{u}$ & WiFi receive power by user $\mathrm{u}$ \\
\hline$p_{I}$ & WiFi interference power \\
\hline$N F$ & noise floor \\
\hline$C_{A v}$ & average user throughput \\
\hline$R$ & WiFi downlink physical transmission rate to users \\
\hline$\varphi$ & link throughput efficiency measured at the IP layer \\
\hline$T$ & $\begin{array}{l}\text { maximum downlink throughput achievable by a LTE } \\
\text { user }\end{array}$ \\
\hline$P L$ & path loss in $\mathrm{dB}$ \\
\hline$d$ & distance in meters \\
\hline NLOS & Non-line-of-sight \\
\hline OF & overbooking factor \\
\hline EIRP & effective isotropic radiated power \\
\hline WF & WiFi First network selection scheme \\
\hline DyBaCS & $\begin{array}{l}\text { Dynamic Backhaul Capacity Sensitive network selection } \\
\text { scheme }\end{array}$ \\
\hline PDR & Physical Data Rate Based network selection scheme \\
\hline MAC & medium access control \\
\hline UE & user equipment \\
\hline$C_{s y s}^{i}$ & system throughput of AP $i$ \\
\hline$C_{\text {sys }}^{\text {LTE }}$ & LTE system throughput within the HetNet \\
\hline$N_{u s e r}^{i}$ & total number of users in AP $i$ \\
\hline$N_{\text {user }}^{\text {LTE }}$ & total number of users in a LTE cell \\
\hline$N_{\text {user }}^{\text {HetNet }}$ & total number of users in HetNet \\
\hline$C_{\text {link,u }}^{i}$ & access link throughput from AP $i$ to user $u$ \\
\hline
\end{tabular}




\begin{tabular}{|c|l|}
\hline$C_{\text {link,u }}^{L T E}$ & access link throughput from LTE BS to user $u$ \\
\hline$C_{b h}^{i}$ & backhaul capacity of AP $i$ in Mbps \\
\hline$C_{A v, l t d}^{i}$ & $\begin{array}{l}\text { average AP } i \text { users' throughput with limited backhaul } \\
\text { capacity }\end{array}$ \\
\hline$C_{A v}^{i}$ & $\begin{array}{l}\text { average AP } i \text { users' throughput with sufficient backhaul } \\
\text { capacity }\end{array}$ \\
\hline$C_{e f f}$ & effective user throughput \\
\hline$C_{n}^{i}$ & estimated throughput if user $n$ is connected to AP $i$ \\
\hline$C_{n}^{L T E}$ & estimated throughput if user $n$ is connected to LTE BS \\
\hline$\Omega$ & $\begin{array}{l}\text { list of higher speed connections (amongst WiFi and LTE } \\
\text { interfaces for a user) for all users in throughput }\end{array}$ \\
\hline$A$ & set that contains all users in the HetNet \\
\hline
\end{tabular}

\subsection{Network Capacity Model}

\subsubsection{Single WiFi AP with Max-min Fairness}

In a multi-rate WiFi network, users with slower link speed tend to occupy more spectrum resource (in terms of transmit time) than higher speed users for the same amount of data sent. Such a bandwidth sharing scenario amongst all users within a single AP coverage has been modelled.

Suppose a total of $N_{\text {user }}$ users are connected to an AP and each is supported by a data rate, $R_{u}$ in accordance to their $S I N R$ level. Thus, user $u$ receives transmission slots at rate $R_{u}$ bps, where $u=1,2 . . N_{\text {user }}$. Assuming the channel access probability is $\mathcal{P}_{\mathrm{u}}$ and it exhibits the effect of slots assignment, which is inversely proportional to user data rate $R_{u}$. Let $S_{u}$ be the number of slots allocated to user $u$, where $S_{u}=k / R_{u}$, with $k$ being an arbitrary constant which will be cancelled off eventually. In this case, if user packet size is assumed to be the same, the AP throughput at the physical layer can be calculated as [25] [26]:

$$
C_{\text {sys }}^{\text {Phy }}=\frac{\sum_{u=1}^{N_{u s e r}} \mathcal{P}_{u} R_{u} S_{u}}{\sum_{u=1}^{N_{u s e r}} \mathcal{P}_{u} S_{u}}
$$

Substituting $S_{u}=\frac{k}{R_{u}}$ into Eqn (1);

$$
C_{\text {sys }}^{\text {Phy }}=\frac{\sum_{u=1}^{N_{u s e r}} \mathcal{P}_{u} R_{u}\left(\frac{k}{R}\right)}{\sum_{u=1}^{N_{u s e r}} \mathcal{P}_{u}\left(\frac{k}{R}\right)}=\frac{k \cdot \sum_{u=1}^{N_{u s e r}} \mathcal{P}_{u}}{k \cdot \sum_{u=1}^{N_{u s e r}} \frac{\mathcal{P}_{u}}{R} u}=\frac{\sum_{u=1}^{N_{u s e r}} \mathcal{P}_{u}}{\sum_{u=1}^{N_{u s e r}} \frac{\mathcal{P}_{u}}{R} u}
$$

Since the sum of $\mathcal{P}_{\mathrm{u}}$ by all user is $\sum_{u=1}^{N_{u s e r}} \mathcal{P}_{u}=1$, Eqn (2) can be further simplified to:

$$
C_{\text {sys }}^{\text {Phy }}=\frac{1}{\sum_{u=1}^{N u s e r} \frac{\mathcal{P}_{u}}{R} u}
$$

The average physical layer throughput per user $C_{A v}^{P h y}$ is calculated by dividing Eqn (3) with the total number of users $N_{\text {user }}$ Since fair resource sharing is assumed, which in turn implies that the packet sending frequency of every user is the same, the $\mathcal{P}_{u}$ of a user can also be written as $\frac{1}{N_{u s e r}}$ :

$$
C_{A v}^{P h y}=\left(\frac{1}{N_{u s e r}}\right) \times\left(\frac{1}{\sum_{u=1}^{N_{u s e r} \frac{1}{N_{u s e r}}}}\right)=\frac{1}{\sum_{u=1}^{N u s e r} \frac{1}{R_{u}}}
$$

The model facilitates max-min fairness [27] [28] behavior in a multi-rate system where all users are allocated an equal amount bandwidth resource. This characteristic is representative of WLAN Distributed Coordinated Function (DCF) [29] where all users are offered a fair medium access opportunity.

Example 1: Assume two users are connected to an AP with supported data rate of $R_{1}=54 \mathrm{Mbps}$ and $R_{2}=6 \mathrm{Mbps}$ respectively.

The number of time slots assigned is inversely proportional to data rate hence $S_{1}=\frac{k}{R_{1}}=\frac{k}{54}$ and $S_{2}=\frac{k}{R_{2}}=\frac{k}{6}$, where $k$ is an arbitrary constant. The channel access probability of user 1 and user 2 are represented by $\mathcal{P}_{1}$ and $\mathcal{P}_{2}$. Since both have a fair chance to transmit to the AP or vice versa, $\mathcal{P}_{1}=\mathcal{P}_{2}=\frac{1}{2}$. With both users taking turn to transmit packets and if their packet sizes are the same, the time taken by each user to transmit a packet can be represented by Figure 2 .

\begin{tabular}{|c|c|}
\hline $\begin{array}{c}\text { User1 } \\
\left(R_{1}=54 \mathrm{Mbps}\right)\end{array}$ & $\begin{array}{c}\text { User2 } \\
\left(R_{2}=6 \mathrm{Mbps}\right)\end{array}$ \\
\hline & $S_{2}=\frac{k}{6}$ \\
\hline
\end{tabular}

Figure 2: Time slots required by user1 and user 2 considering fair throughput

From Figure 2, User 2 takes more time than User 1 in order to maintain throughput fairness. In other words, User 1 is being penalized in terms of time slot fairness, which is the characteristic of WiFi DCF system. The average throughput for both users can be calculated using Eqn (5) as follows:

$$
C_{A V}^{P h y}=\frac{S_{1}}{S_{1}+S_{2}} \times R_{1}=\frac{S_{2}}{S_{1}+S_{2}} \times R_{2}
$$

By substituting the values of $S_{1}, S_{2}, R_{1}$ and $R_{2}$ into Eqn (5), the average throughput for both users can be calculated as 5.4 Mbps.

Similarly, within a single WiFi network, the average IP layer throughput per user $C_{A v}$ (Eqn (6)) is calculated by multiplying the physical data rate $R_{u}$ in Eqn (4) by a corresponding throughput efficiency factor $\varphi_{u}$ given in TABLE V:

$$
C_{A v}=\frac{1}{\sum_{u=1}^{N u s e r} \frac{1}{R u \times \varphi_{u}}}
$$

The accuracy of the model (implementation of Eqn (6)) is verified together with the IEEE802.11g WiFi models (in 
section 3.1) using the QualNet event-driven simulator (Version 5.2) and the results are found to be very similar.

\subsubsection{LTE with Max-Min Fairness}

In LTE, resource is assigned to users in forms of Physical Resource Blocks (PRB) consisting of many Resource Elements. The same max-min fair bandwidth sharing approach is adopted where in the LTE case, more PRBs are assigned to users experiencing worse channel behavior to ensure fairness. This assumption is reasonable since the LTE Medium Access Control (MAC) scheduler has not been fully defined in the standards. In order to simplify the study, resource element allocations in LTE are approximated as time fraction allocation in a TDMA manner as modeled in [16] and the average user throughput is defined by Eqn (7), a straightforward modification from Eqn (6):

$$
C_{A v}=\frac{1}{\sum_{u=1}^{N N_{u S E} \frac{1}{T_{u}}}}
$$

where $T_{u}$ is maximum link throughput achievable by LTE user $u$ and $N_{u s e r}^{L T E}$ is the total number of LTE users.

\subsection{Network Selection Schemes}

In a heterogeneous wireless network comprising two or more technologies, user throughput fairness needs to be considered in two aspects, intra-network fairness and internetwork fairness. Intra-network fairness can be provided by AP or LTE BS to its associated users as the task is confined locally. With max-min fair capacity sharing of WiFi and LTE network (Eqn (6) and Eqn (7)), fairness is achievable amongst users under the same AP or BS. However, fair capacity sharing across the entire HetNet is not guaranteed and is strongly dependent on the implemented NSSs. The proposed DyBaCS is compared with two commonly used NSSs namely WiFi First (WF) and Physical Data Rate (PDR), the characteristics of which are as follows:

\subsubsection{WiFi First (WF)}

WiFi First (WF) connects a user to an AP whenever WiFi coverage is available. In other words, a user will never connect to a LTE network when there is WiFi coverage. This approach is adopted by most smart phones' and tablets' connection manager by default as the preferred data connection mode. In addition, this mode is also promoted by some mobile operators as a means to offload traffic from mobile networks [30] [7].

\subsubsection{Physical Data Rate (PDR)}

PDR chooses the network based on the Physical data rate of the RAT available to the user [8] [9]. Here the physical data rates provided by LTE and WiFi are compared and the RAT with higher physical data rate is chosen. Since PDR tends to assign more bandwidth to the users with better link capacity, it compromises user fairness, though the overall throughput performance might be good.

\subsubsection{Proposed Dynamic Backhaul Capacity Sensitive (DyBaCS) scheme}

DyBaCS is mainly proposed to preserve fairness while maximizing HetNet throughput. Thus DyBaCS throughput performance may not outperform PDR (which is optimum in maintaining HetNet throughput), but it is very close to PDR in most cases (Section 4). In the study, a single LTE network is assumed with $I(I \geq 1)$ denoting the total number of $\mathrm{WiFi}$ APs in the HetNet and $i$ denoting the index of the network, i.e. network $i=1 \leq i \leq I$ is the $i$-th WiFi network, while, $u=1 \leq u \leq N_{\text {user }}^{\text {Het }}$ ret represents the $u$-th user in the HetNet and $U$ is the total user in entire HetNet. Total number of users in AP $i$ and LTE network is represented by $U^{i}$ and $U^{L T E}$ respectively.

Before explaining the DyBaCS algorithm in detail, Algorithm 1; the User Throughput Estimation Flow (UTEF) algorithm, which enables the estimation of $\mathrm{WiFi}$ user throughputs $C_{e f f, u}^{i}$ and LTE user throughputs $C_{e f f, u}^{L T E}$ is first presented as follows:

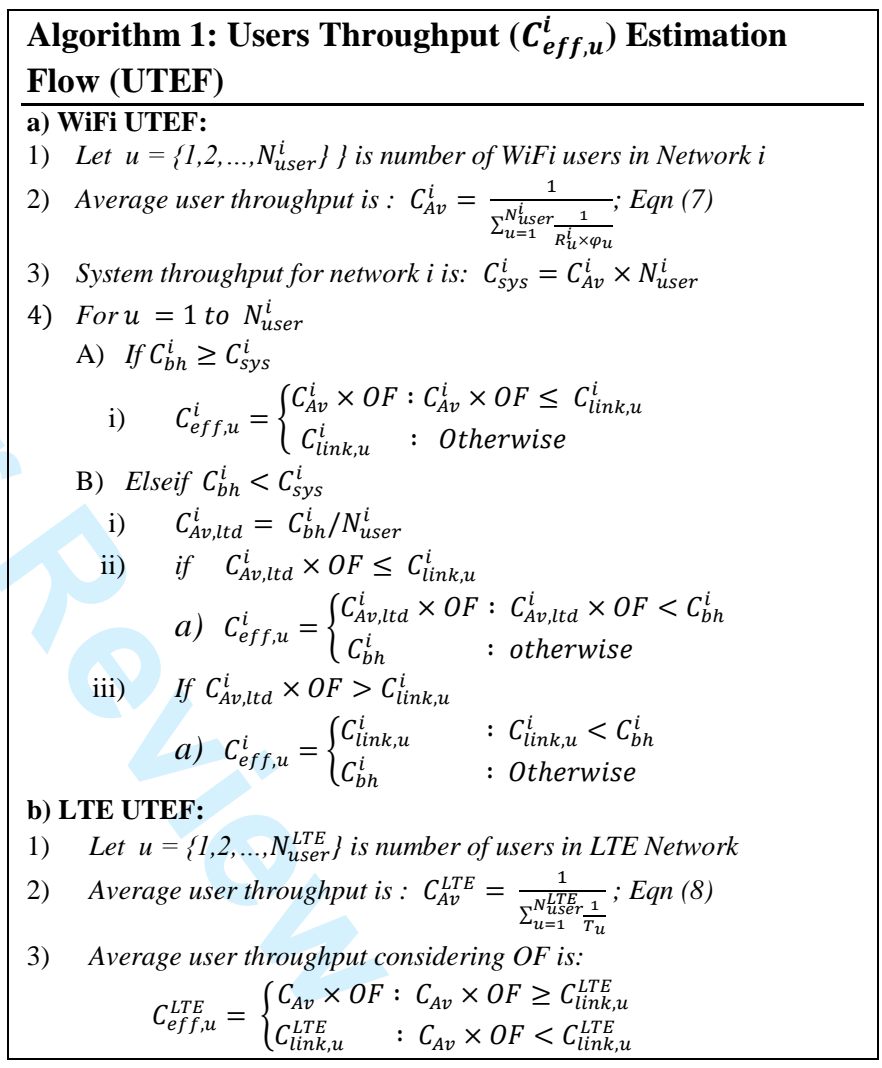

For WiFi UTEF (Algorithm 1), the calculation of effective WiFi user throughput $C_{e f f, u}^{i}$ is based on user access link throughput $C_{\text {link,u }}^{i}$, AP backhaul capacity $C_{b h}^{i}$ and Overbooking Factor (OF). Link throughput $C_{\text {link,u }}^{i}$ (or equivalent to $R_{u}^{i} \times \varphi_{u}$ in Eqn (6) and $\varphi_{u}$ is listed in TABLE V) of user $u$ is predominately affected by the distance from the AP, channel quality and other channel conditions such as interference. Backhaul capacity $C_{b h}^{i}$ is defined as the actual available backhaul capacity to $\mathrm{AP} i$, whether it is wired or wireless backhaul. Effective user throughput is limited by both 
factors. In practice, OF is normally considered as not all the users access the channel at the same time [31]; by including OF the perceived throughput from the users point of view can be calculated.

The calculation of effective user throughput in WiFi UETF can be explained in detail with the aid of the scenario shown in Figure 3, where 4 users are connected to AP $i$. User data rate $R_{u}^{i}$ and link throughput $C_{\text {link }, u}^{i}$ are listed in TABLE II.

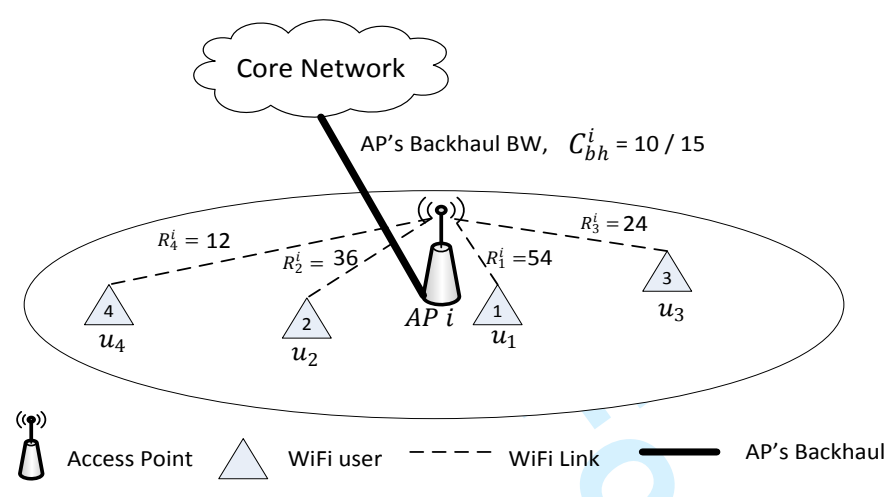

Figure 3: AP with 4 users under its coverage

TABLE II : USER DATA RATE AND LINK THROUGHPUT IN Figure 3

\begin{tabular}{ccc}
\hline User, $\mathbf{u}$ & $\boldsymbol{R}_{\boldsymbol{u}}^{\boldsymbol{i}}$ & $\boldsymbol{C}_{\boldsymbol{l i n} \boldsymbol{k}, \boldsymbol{u}}^{\boldsymbol{i}}=\boldsymbol{R}_{\boldsymbol{u}}^{\boldsymbol{i}} \times \boldsymbol{\varphi}_{\boldsymbol{u}}$ \\
\hline 1 & 54 & $54 \times 0.32=17.28$ \\
2 & 36 & $36 \times 0.41=14.76$ \\
3 & 24 & $24 \times 0.49=11.76$ \\
4 & 12 & $12 \times 0.61=7.32$ \\
\hline
\end{tabular}

Step 1 and Step 2 in Algorithm 1 estimate the average WiFi user throughput using Eqn (6) and the average user throughput can be calculated as:

$$
\begin{aligned}
C_{A v}^{i}= & \frac{1}{\sum_{u=1}^{N_{u s e r}^{i}} \frac{1}{R_{u}^{i} \times \varphi_{u}}}=\frac{1}{\frac{1}{R_{1}^{i} \times \varphi_{1}}+\frac{1}{R_{2}^{i} \times \varphi_{2}}+\frac{1}{R_{3}^{i} \times \varphi_{3}}+\frac{1}{R_{4}^{i} \times \varphi_{4}}} \\
= & \frac{1}{54 \times 0.32}+\frac{1}{36 \times 0.41}+\frac{1}{24 \times 0.49}+\frac{1}{12 \times 0.61} \\
& =2.88 \mathrm{M}
\end{aligned}
$$

Multiplying the average user throughput $C_{A v}^{i}$ by the total number of users $N_{u s e r}^{i}$, as in Step 3, yields the total system throughput $C_{s y s}^{i}$ of AP $i$ :

$$
C_{s y s}^{i}=C_{A v}^{i} \times N_{u s e r}^{i}
$$

In the scenario, $C_{s y s}^{i}=2.88 \times 4=11.52 \mathrm{Mbps}$. As in Eqn (6), $C_{s y s}^{i}$ is affected by link speed $R_{u}$ of all users in network $i$; therefore it is highly dependent on users distribution. $C_{s y s}^{i}$ represents the required backhaul capacity for AP $i$ to realise its full access capacity. If the backhaul capacity is sufficient to support the maximum capacity from the AP to clients such that $C_{b h}^{i} \geq C_{s y s}^{i}$, then all users will enjoy a throughput of $C_{A v}^{i}$. However, if the backhaul capacity $C_{b h}^{i}$ is the bottleneck, $C_{b h}^{i} \leq C_{s y s}^{i}$, the available backhaul capacity $C_{b h}^{i}$ is shared evenly to all users $N_{u s e r}^{i}$ and average user throughput in such scenario is limited to a value $C_{A v, l t d}^{i}$ (Eqn (10)) which is less than $C_{A v}^{i}$.

$$
C_{A v, l t d}^{i}=C_{b h}^{i} / N_{u s e r}^{i}
$$

It is important to note that users will not secure a throughput higher than $C_{A v}^{i}$ even at backhaul capacities greater than $C_{s y s}^{i}$ due to the restriction imposed by the WiFi physical and MAC layer capability. Under the assumption of simultaneous channel access, $C_{A v}^{i}$ or $C_{A v, l t d}^{i}$ represents the average user throughput for both sufficient and limited backhaul capacity cases.

In Figure 3, assuming that backhaul capacity $C_{b h}^{i}=10$ is less than $C_{s y s}^{i}=11.52$, the actual average throughput for the users is limited to $C_{A v, l t d}^{i}=\frac{C_{b h}^{i}}{N_{u s e r}^{i}}=\frac{10}{4}=2.5 \quad$ Mbps. Otherwise, each user will enjoy $C_{A v}^{i}=2.88 \mathrm{Mbps}$ if $C_{b h}^{i} \geq$ $C_{s y s}^{i}$.

In order to simplify the explanation of Algorithm 1, an OF $=5$ is adopted for scenario depicted in Figure 3. Suppose $C_{b h}^{i}=$ 15 Mbps where $C_{b h}^{i} \geq C_{s y s}^{i}$, the effective average throughput $C_{e f f, u}^{i}$ of user $u$ is only limited by its link throughput $C_{\text {link }, u}^{i}$. Step $4 \mathrm{~A}$ (i) places a constraint to ensure that the value of $C_{A v}^{i} \times O F$ does not exceed $C_{\text {link }, u}^{i}$. TABLE III shows that effective user throughput $C_{e f f, u}^{i}$ is the minimum value between $C_{A v}^{i} \times O F$ and $C_{\text {link,u }}^{i}$. Note that the effective average throughput for User 3 and User 4 is limited to their link throughput which is $11.76 \mathrm{Mbps}$ and 7.32 Mbps respectively.

TABLE III: EFFECTIVE USER THEROUGHPUT $C_{e f f, u}^{i}$ UNDER CONDITION $C_{b h}^{i} \geq C_{s y s}^{i}$.

\begin{tabular}{cccccc}
\hline User, $\boldsymbol{u}$ & $\boldsymbol{C}_{\boldsymbol{A \nu}}^{\boldsymbol{i}}$ & $\boldsymbol{C}_{\boldsymbol{b h}}^{\boldsymbol{i}}$ & $\boldsymbol{C}_{\text {link, } \boldsymbol{u}}^{\boldsymbol{i}}$ & $\boldsymbol{C}_{\boldsymbol{A} \boldsymbol{v}}^{\boldsymbol{i}} \times \boldsymbol{O F}$ & $\boldsymbol{C}_{\boldsymbol{e f f}, \boldsymbol{u}}^{\boldsymbol{i}}$ \\
\hline 1 & 2.88 & 15 & 17.28 & 14.40 & 14.40 \\
2 & 2.88 & 15 & 14.76 & 14.40 & 14.40 \\
3 & 2.88 & 15 & 11.76 & 14.40 & 11.76 \\
4 & 2.88 & 15 & 7.32 & 14.40 & 7.32 \\
\hline
\end{tabular}

Likewise, with limited backhaul capacity, Step 4B (ii) and Step 4B (iii) ensure that $C_{A v, l t d}^{i} \times O F$ is bound by both $C_{\text {link, } u}^{i}$ and $C_{b h}^{i}$, whichever is smaller. The result is shown in TABLE IV.

TABLE IV : EFFECTIVE USER THROUGHPUT $C_{e f f, u}^{i}$ UNDER CONDITION $C_{b h}^{i} \leq C_{s y s}^{i}$.

\begin{tabular}{cccccc}
\hline User, $\boldsymbol{u}$ & $\boldsymbol{C}_{\boldsymbol{A} \boldsymbol{v}}^{\boldsymbol{i}}$ & $\boldsymbol{C}_{\boldsymbol{b h}}^{\boldsymbol{i}}$ & $\boldsymbol{C}_{\text {lin } \boldsymbol{k}, \boldsymbol{u}}^{\boldsymbol{i}}$ & $\boldsymbol{C}_{\boldsymbol{A} \boldsymbol{v}}^{\boldsymbol{i}} \times \boldsymbol{O F}$ & $\boldsymbol{C}_{\boldsymbol{e f f}, \boldsymbol{u}}^{\boldsymbol{i}}$ \\
\hline 1 & 2.88 & 10 & 17.28 & 12.5 & 10 \\
2 & 2.88 & 10 & 14.76 & 12.5 & 10 \\
3 & 2.88 & 10 & 11.76 & 12.5 & 10 \\
4 & 2.88 & 10 & 7.32 & 12.5 & 7.32 \\
\hline
\end{tabular}


In short, Step 4 imposes logical considerations to the determination of the effective user average throughput $C_{e f f, u}^{i}$ taking into consideration backhaul capacity, user link speed and OF. Similarly, the LTE user throughput can also be estimated using the LTE UTEF approach. In this paper, LTE backhaul capacity is always assumed to be sufficient. Therefore in Step 3, the only constraint for user throughput in the LTE network is the access link throughput $C_{\text {link, } u}^{L T E}$.

The DyBaCS NSS (Algorithm 2) assumes that initially, there is no user connected to the HetNet. Users are admitted to the HetNet one by one and their servicing network is determined by the NSS.

Step1 initializes variables $\Omega, A, N_{\text {user }}^{i}$ and $N_{\text {user }}^{L T E}$, where $\Omega$ is a list holding the higher speed connections between $\mathrm{WiFi}$ and LTE interfaces to users, $A$ is the set containing all users in the HetNet, $N_{\text {user }}^{i}$ is the list of users connected to the WiFi AP $i$ and $N_{\text {user }}^{L T E}$ is the list of users connected to the LTE network.

In Step 2, the access link with the highest throughput between user $u$ to $\operatorname{LTE}\left(C_{\text {link }, u}^{L T E}\right)$ or $\mathrm{WiFi}\left(C_{\text {link,u }}^{i}\right)$ is chosen and placed into $\Omega, \Omega_{u}$ represents the throughput value for user $u$. All users are able to connect to at least the LTE network while access to $\mathrm{WiFi}$ depends on availability of $\mathrm{WiFi}$ coverage. Hence $\beta_{u}^{i}=\{0,1\}$ denotes the connectivity of user $u$ to $\mathrm{AP} i, \beta_{u}^{i}=$ ' 1 ' means connection is possible, '0' means the opposite. Since multi-homing is not considered, every user is only allowed to connect to one network at a time. Step 3 assigns all users with no $\mathrm{WiFi}$ access to the LTE network and excludes those users from set $A$. The network selection parameter $\alpha_{u}^{i} \in\{0,1\}$ indicates the choice of user $u ; \alpha_{u}^{i}={ }^{\prime} 1$ ', indicates that user $u$ is connected to network $i$ and value ' 0 ' means the opposite.

The remaining users within set $A$ which have not been assigned to any network are subjected to network selection; they can either assigned to $\mathrm{WiFi}$ or LTE. Network selection for those users is addressed in Step 4 in order to optimize the network performance. Step 4 (a) ensures the user with the highest link throughput within set $A$ (referred to as user $n$ ) is considered first.

In Step 4 (b) and Step 4 (c), the achievable throughput of user $n$ (considering access link speed, AP backhaul capacity and $\mathrm{OF}$ ) on WiFi AP $i$ and LTE is estimated using WiFi UTEF and LTE UTEF and represented by $C_{e f f, n}^{i}$ and $C_{e f f, n}^{L T E}$ respectively. The throughput estimation is executed assuming that user $n$ is added to the corresponding WiFi or LTE network. Therefore, during the throughput estimation, the total number of users connected to AP $i$ is assumed to be user $n$ plus the total number of existing users $N_{u s e r}^{i}$ written as $N_{\text {user }}^{i} \cup\{n\}$ and similarly the total number of users in the LTE network is $N_{\text {user }}^{L T E} \cup\{n\}$. The achievable throughput for user $n$ on the $\mathrm{WiFi}$ and LTE network is then assigned to $C_{n}^{i}$ and $C_{n}^{L T E}$ respectively.

User $n$ is subsequently assigned to the network that offers the highest throughput and the corresponding $\alpha_{n}^{L T E}$ or $\alpha_{n}^{i}$ value is set according to Step 4 (d). The total number of users connected to their respective $i$-th $\mathrm{WiFi}$ network $N_{\text {user }}^{i}$ or LTE network $N_{\text {user }}^{L T E}$ is then updated.

Finally set $A$ is updated by excluding user $n$ in Step 4(e) and the Step 4 processes are repeated until all users are serviced.

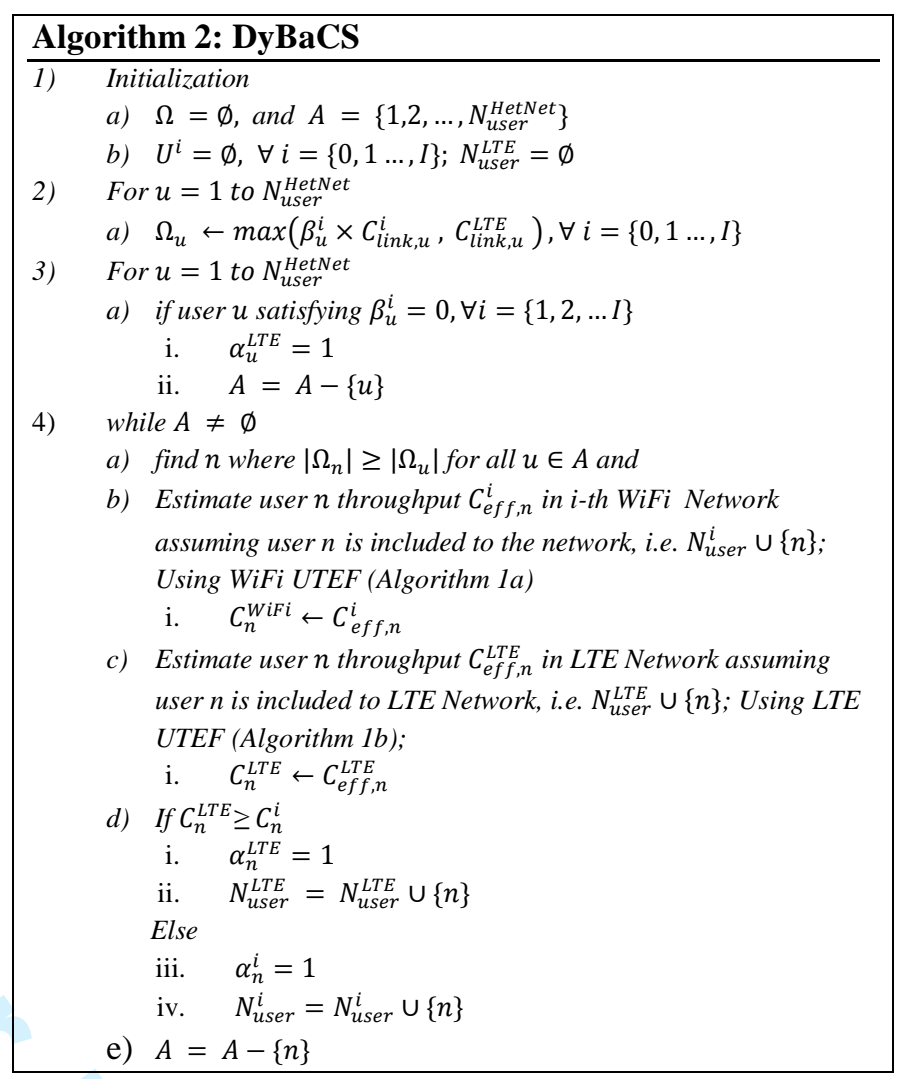

\section{Parameters, Assumptions and Simulation Approach}

The section describes the simulation approach, simulation parameters and assumptions used.

\subsection{WiFi Model}

TABLE $\mathrm{V}$ presents the parameters used in the $\mathrm{WiFi}$ IEEE802.11g model. The receiver sensitivity values per modulation and coding scheme (MCS) for $\mathrm{WiFi}$ receivers are taken from [32]. The throughput efficiency, the ratio of actual IP layer throughput against physical data rate and the actual IP layer throughput of each MCS scheme are obtained from the QualNet simulator assuming fixed packet size of 1000 Bytes with constant bit rate traffic.

TABLE V : IEEE802.11G PARAMETERS

\begin{tabular}{cccccc}
\hline Index & MCS & $\begin{array}{c}\text { Receiver } \\
\text { Sensitivity } \\
(\mathbf{d B m})\end{array}$ & $\begin{array}{c}\text { Data } \\
\text { Rate } \\
(\mathbf{M b p s})\end{array}$ & $\begin{array}{c}\text { Throughput } \\
\text { Efficiency } \\
(\boldsymbol{\varphi})\end{array}$ & $\begin{array}{c}\text { Throughput } \\
(\mathbf{M b p s})\end{array}$ \\
\hline 1 & BPSK1/2 & -94.0 & 6 & 0.70 & 4.20 \\
2 & BPSK3/4 & -93.0 & 9 & 0.64 & 5.76 \\
3 & QPSK1/2 & -91.0 & 12 & 0.61 & 7.32 \\
4 & QPSK3/4 & -90.0 & 18 & 0.54 & 9.72 \\
5 & 16-QAM1/2 & -86.0 & 24 & 0.49 & 11.76 \\
6 & 16-QAM3/4 & -83.0 & 36 & 0.41 & 14.76 \\
7 & 64-QAM2/3 & -77.0 & 48 & 0.35 & 16.80 \\
8 & 64-QAM3/4 & -74.0 & 54 & 0.32 & 17.28 \\
\hline
\end{tabular}


The path loss model in Eqn (11) is derived from field measurement detailed in [33] :

$$
P L=30.2 * \log 10(d)+22.234
$$

\subsection{LTE Model}

LTE throughput is calculated based on the throughput plot by 3GPP [34] shown in Figure 4. In the simulation, SINR ratio at a receiver is used to calculate the achievable throughput. In order to calculate the received power, the ITU recommended LTE Urban Macro (UMa) non-line-of-sight (NLOS) path loss model is used [35].

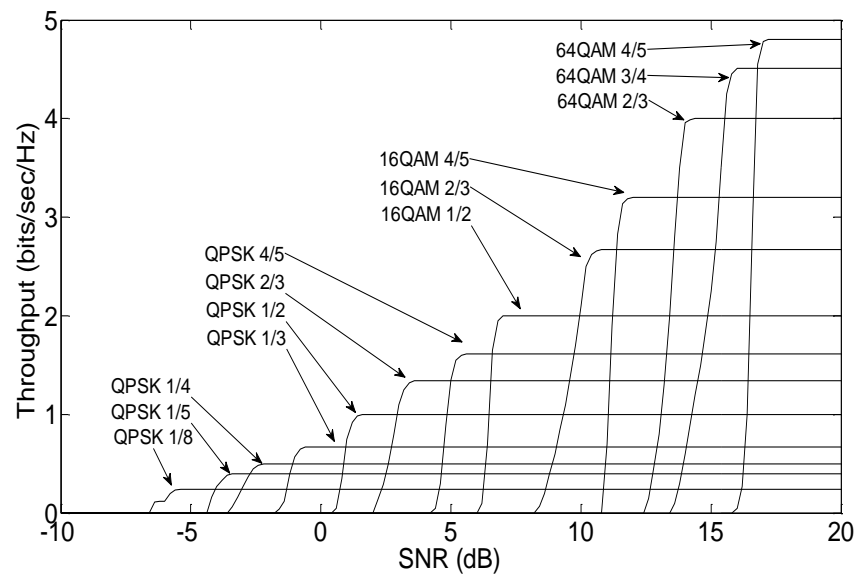

Figure 4: LTE throughput vs. SNR for different Modulation and Coding Schemes (MCS) used in the model

\subsection{Stochastic User Placement Model}

A stochastic node location model is used to position the users' in the HetNet. The authors in [36] found that this model can represent the users' locations around WiFi AP in a city or urban environment. Here users are assumed to be distributed around cluster centers. Two spatial distributions model are adopted where the first distribution is used to generate the numbers and locations of the cluster centers. The second distribution is then used for positioning the number of users around each center. The initial cluster center distribution is generated randomly with total number of cluster centers equal to $N_{\text {cluster }}$. Subsequently, a bivariate Gaussian distribution with covariance matrix $\sum=\operatorname{diag}\left(\sigma_{x}^{2}, \sigma_{y}^{2}\right)$ is used to generate actual user locations around the cluster centres. The size and shape of the cluster is characterized by the parameters $\sigma_{x}$ and $\sigma_{y}$ (Figure 5). The number of users per cluster is drawn from a Poisson distribution with intensity $N_{\text {user }}$. Parameters $\sigma_{x}, \sigma_{y}$ and $N_{\text {user }}$ are the same for every cluster.

In the simulation, initially 100 users are placed in an area of 1 square kilometre with number of clusters $N_{\text {cluster }}=10$, cluster size $N_{\text {user }}=10$ and $\sigma_{x}=\sigma_{y}=25$. Subsequently 50 users are added each time by increasing number of cluster $N_{\text {cluster }}$ by five while keeping the number of users per cluster $N_{\text {user }}$ and cluster dimension $\sigma$ unchanged, until the total number of users reaches 250 (Figure 6). Since all users are tabulated in an area of 1 square kilometre, the terms the number of users and user density are used interchangeably in the rest of this paper.

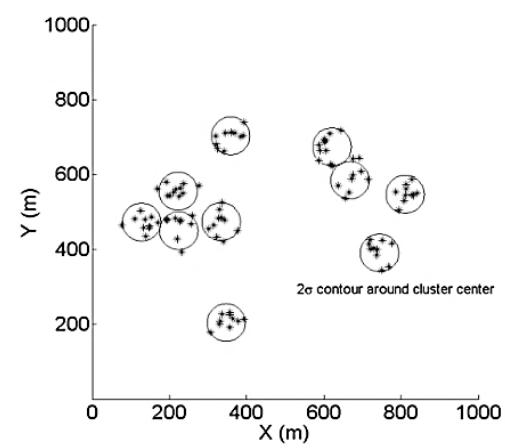

Figure 5: User placement with total number of clusters $N_{\text {cluster }}=10$, cluster size $N_{\text {user }}=10$ and $\sigma=25$

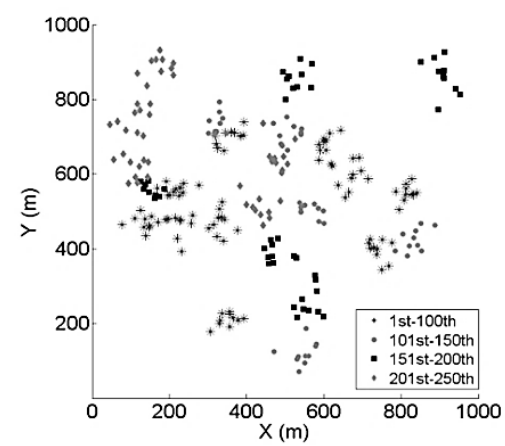

Figure 6: User placement from 100 users to 250 users with 50 users added every time, maximum $N_{\text {cluster }}=25$, cluster size $N_{\text {user }}=10$ and $\sigma=25$

\subsection{Placement of WiFi Access Points}
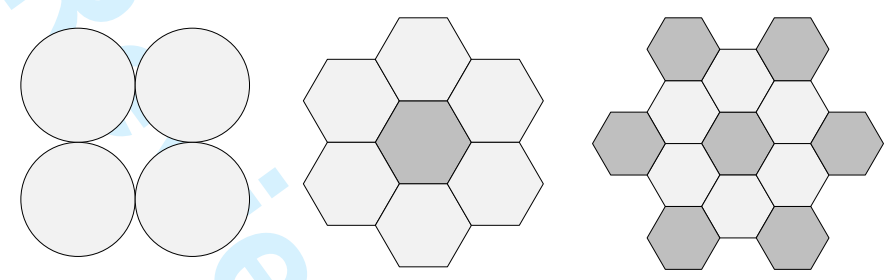

Figure 7: WiFi APs placement within HetNet that comprises the scenarios with 4, 7 and $13 \mathrm{WiFi}$ APs

In the Matlab model, WiFi APs are placed within a macro LTE cell according to three selected topologies shown in Figure 7. These WiFi topologies enable the NSSs to be evaluated as a function of WiFi-LTE node ratios (or WiFi node densities). By controlling the APs' transmit power, these WiFi topologies can give the same coverage size as the macro LTE cell. For example, Figure 8 a shows the coverage plot for 4 APs, while the overlay of both WiFi and LTE cell (Figure 8b) that forms the HetNet is illustrated in Figure 8c. WiFi coverage plots for 7 and 13 APs are shown in Figure 9a and Figure 9b respectively. Although there is no limit on the variation topologies which can be evaluated, the selected topologies is believed to be able to provide a good insight on the performance of NSSs under different WiFi-LTE node ratios. 




(a)



(c)

Figure 8: Network coverage plot based on data rate with $\mathrm{x}$ and $\mathrm{y}$ axis showing coverage size in meter; a) 4-WiFi-Nodes networks with access channel shown in parentheses, b) LTE network and c) HetNet (LTE+WiFi) with 100 users

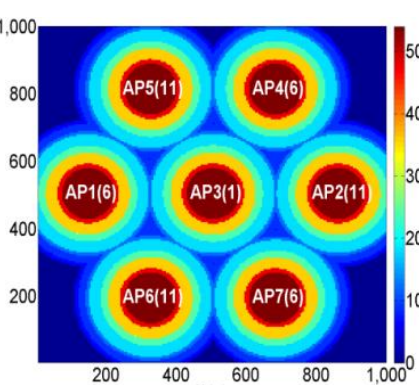

(a)

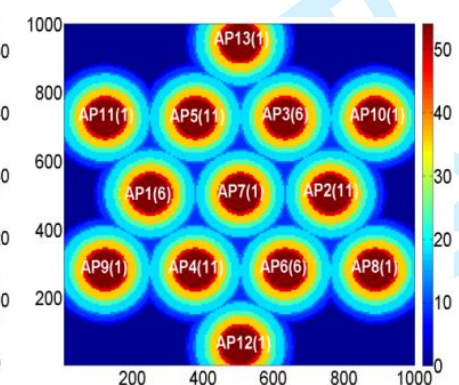

(b)
Figure 9: WiFi coverage plot based on data rate with $\mathrm{x}$ and $\mathrm{y}$ axis showing coverage size in meter; a) 7-WiFi-Nodes networks, b) 13-WiFi-Nodes networks; WiFi access channel shown in parentheses

\subsection{WiFi Access Channel and Interference}

On WiFi access channel, three non-overlapping channels with channel number 1, 6 and 11 are used (channel number is stated on top of each AP in Figure 8 and Figure 9). To calculate SINR, the simulated area is divided into 5-by-5 square meter grids. Since non-overlapping channels are used, only cochannel interference is considered. In each grid, an effective SINR is derived as:

$$
\operatorname{SINR}=\frac{p_{u}}{\sum_{t=1}^{M} p_{I}+N F_{i}}
$$

where, $p_{u}$ is the received power of user $u$ from the serving AP located in a grid, $M$ is total number of co-channel interferer APs in range, $p_{I}$ is the interference power at the receiver and $N F_{u}$ is the noise floor. Effective SINR obtained is then used to map user data rate and hence user throughput calculation in WiFi networks.

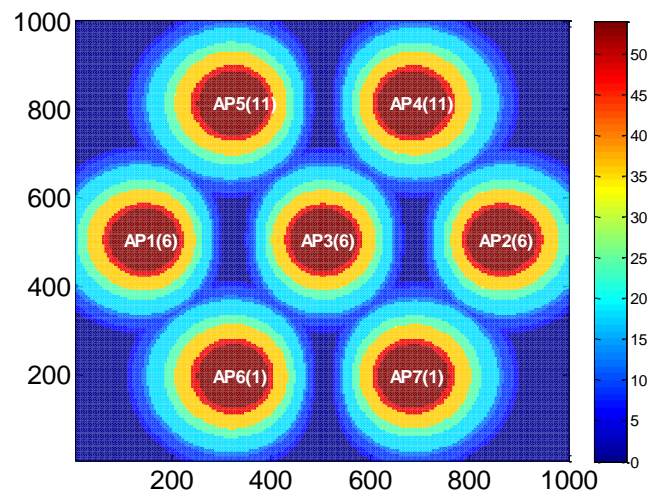

Figure 10: 7-WiFi-Nodes coverage plot with co-channel interference

Figure 10 shows the coverage plot of $7 \mathrm{WiFi}$ nodes with cochannel interference. The interference has resulted in a smaller effective WiFi coverage area as compared to Figure 9a, which has a better channel plan.

\subsection{WiFi and LTE backhaul}

In the study, WiFi backhaul capacity is varied from $1 \mathrm{Mbps}$ up to $25 \mathrm{Mbps}$ until the throughput of AP saturates (Section 4). Two backhaul scenarios are assumed when evaluating the NSSs; 1) uniform WiFi backhaul capacity and 2) non-uniform WiFi backhaul capacity throughout the HetNet. The LTE base station (BS) backhaul capacity is always assumed to be sufficient.

\subsection{General Parameters and Assumptions}

TABLE VI summarizes the simulation parameters used. Only the downlink performance is investigated in the paper. $2.6 \mathrm{GHz}$ is used for LTE as it is the frequency band allocated in Malaysia. A fixed interference margin of $3 \mathrm{~dB}$ is assumed for the LTE User Equipment (UE).

TABLE VI : WIFI AND LTE SIMULATION PARAMETERS

\begin{tabular}{cccc}
\hline & Units & WiFi 11g & LTE \\
\hline Frequency band & $\mathrm{GHz}$ & 2.4 & 2.6 \\
Channel bandwidth & $\mathrm{MHz}$ & 20 & 20 \\
Max EIRP & $\mathrm{dBm}$ & $27,24,18$ & 36 \\
Rx antenna gain & $\mathrm{dBi}$ & 3 & 0 \\
Antenna diversity gain & $\mathrm{dB}$ & 3 & 3 \\
Noise Figure & $\mathrm{dB}$ & Not required & 10 \\
Packet size & Bytes & 1000 \\
WiFi backhaul capacity & Mbps & $1-25$ \\
LTE backhaul capacity & Mbps & Sufficient capacity assumed \\
\hline \multicolumn{4}{c}{} \\
\hline Overbooking Factor, $O F$ & User Information \\
User density & - & \multicolumn{3}{c}{$10: 1$} \\
\end{tabular}

Receiver antenna gain of WiFi and LTE UE is assumed to be $3 \mathrm{~dB}$ and $0 \mathrm{~dB}$ respectively. The assumption on traffic type has been described in Section 3.3.1. From [31], the Overbooking Factor (OF) is a design choice driven by actual user behavior in the deployed area. OF that varies from $4: 1$ to 100:1 has been reported by various ISPs and the lower the 


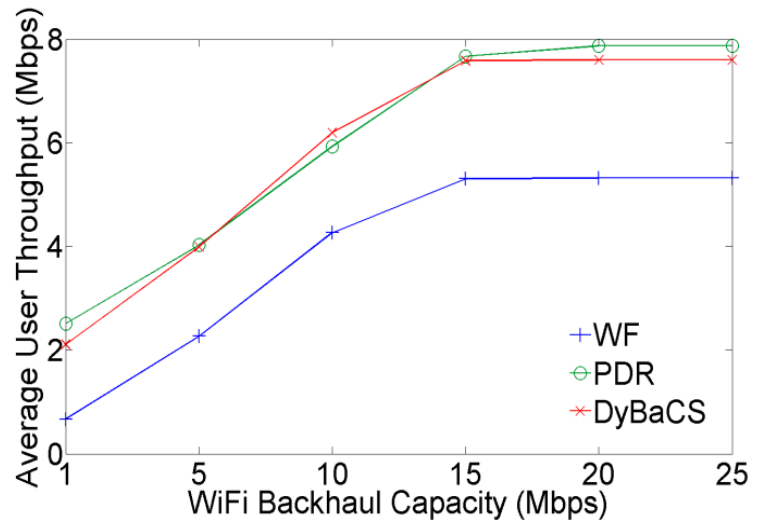

Figure 11: HetNet average user throughput as a function of WiFi backhaul capacity. (4 WiFi APs, 100\% WiFi-LTE overlap, Uniform WiFi backhaul capacity)

value the better the service guarantee. A factor of 10:1 in adopted to represent a relatively heavy usage scenario. User density is varied from 100 users to 250 users per square $\mathrm{km}$.

\section{Results and Analysis}

\subsection{Effect of Varying Backhaul Capacity Uniformly}

Two performance metrics namely average user throughput and user throughput fairness in the HetNet cell are used to evaluate the performance of the NSSs. Fairness is derived using Jain fairness index in the form of $\left(\sum_{u=1}^{N_{u s e r}} C_{e f f, u}\right)^{2}$ / $\left(N_{u s e r} \sum_{u=1}^{N_{u s e r}}\left(C_{e f f, u}\right)^{2}\right)$ [37].

First the performance of all NSSs is evaluated as a function of WiFi backhaul capacity from $1 \mathrm{Mbps}$ to $25 \mathrm{Mbps}$ with $100 \%$ WiFi-LTE coverage overlap and 4 WiFi APs (as in Figure 8c). Figure 11 shows that average user throughput of PDR and DyBaCS are similar over the entire range of backhaul capacity and WF has the lowest average user throughput. The plots plateaus when backhaul capacity reaches 20Mbps for all NSSs as that is the maximum access throughput achievable by WiFi (IEEE 802.11g). The total $\mathrm{WiFi}$ access throughput also depends on user distribution as slower users slow down the entire network. Figure 12 evaluates the fairness in terms of achievable throughput per user. Result shows that average user throughput of PDR and DyBaCS are similar over the entire range of backhaul capacity while WF has the lowest average user throughput. DyBaCS outperforms the rest in terms of fairness and WF exhibits the worst fairness performance.

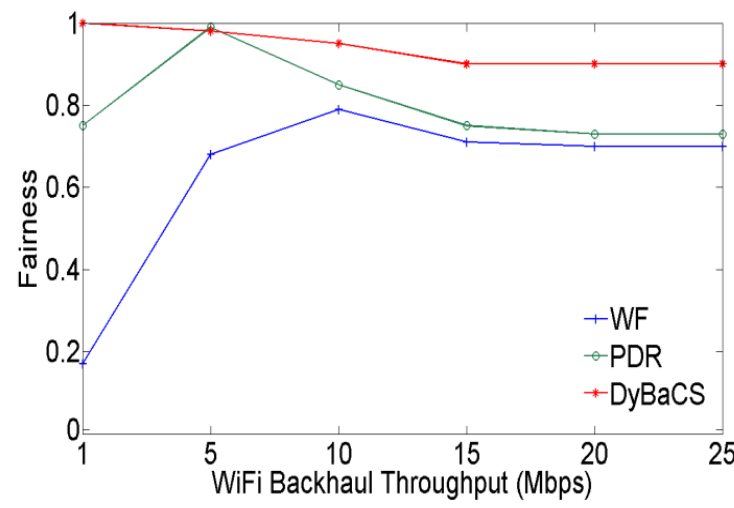

Figure 12: Fairness on bandwidth sharing as a function of WiFi backhaul capacity. (4 WiFi APs, 100\% WiFi-LTE overlap, Uniform WiFi backhaul capacity)

\subsection{Effect of WiFi-LTE Overlap Ratio Within a HetNet cell}

Since the coverage overlap between WiFi and LTE is likely to vary in practice, it is therefore important to investigate how NSSs perform in different overlap conditions. Here the coverage area is defined as a percentage e.g. $25 \%$ means $25 \%$ of LTE coverage is also covered by $\mathrm{WiFi}$ and $100 \%$ means all LTE coverage is overlapped by WiFi. The simulation is carried out by switching on APs within the LTE cell one by one until the entire LTE cell overlaps with WiFi coverage. AP activation sequence is based on the number of users covers by an AP, AP which covers the most users is switched on first and followed by APs with lesser users in descending order.

Figure 13a shows that in general, that the average user throughput increases linearly for all NSSs as backhaul capacity increases and starting to flatten at $15 \mathrm{Mbps}$. The maximum average user throughput for PDR, DyBaCS and WF are 7.87Mbps, $7.60 \mathrm{Mbps}$ and $5.32 \mathrm{Mbps}$ respectively at $100 \%$ WiFi-LTE coverage overlap and 20Mbps backhaul capacity. PDR which has the highest average throughput only has a marginal performance edge as compared to DyBaCS. The difference in performances diminishes when WiFi-LTE coverage overlap increases. WF provides the worst average user throughput in most cases. An interesting point to note is for WF at $1 \mathrm{Mbps}$ backhaul capacity, increases in $\mathrm{WiFi}$ coverage results in a drop in the average user throughput instead of increase. This is due to the fact that WF connects all users to WiFi networks regardless of its backhaul capacity. Consequently, with limited WiFi backhaul capacity as low as $1 \mathrm{Mbps}$, WF causes the users to suffer from low average throughput and becoming more severe when $\mathrm{WiFi}$ coverage is larger. 


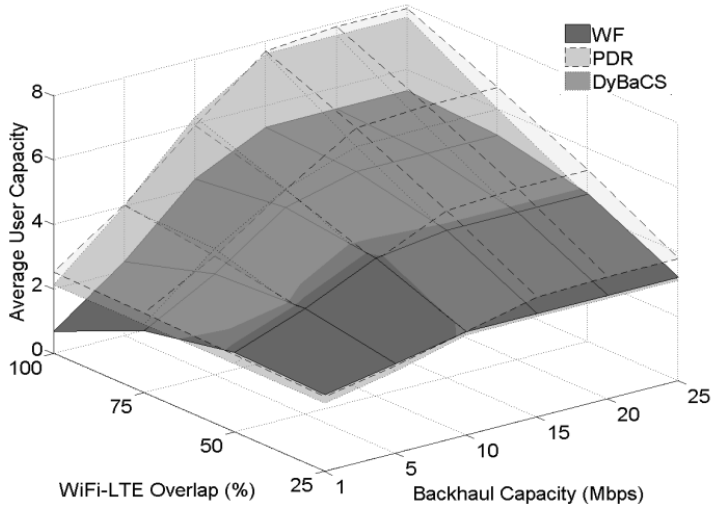

(a) Average User Throughput



(b) Fairness

Figure 13: NSSs performance as a function of backhaul capacity and WiFi-LTE overlap percentage (Maximum 4 WiFi APs, Uniform WiFi backhaul capacity).

Figure $13 \mathrm{~b}$ shows that DyBaCS has the highest fairness for the entire range of $\mathrm{WiFi}$-LTE overlap ratio and backhaul capacity considered. As for WF, higher fairness can be achieved with higher WiFi backhaul capacity because adequate capacity is shared amongst users. It is also found that fairness of PDR is closer to DyCaBS when backhaul capacity is low but the difference between them becomes larger as the backhaul capacity increases because the capacity distribution of PDR is less fair. At 25Mbps backhaul capacity and $100 \%$ WiFi-LTE coverage overlap, both WF and PDR achieve 0.7 and 0.73 respectively in terms of fairness, but this is lower than 0.9 by DyBaCS.

\subsection{Effect of User Density}

A high probability that the number of users in a network will vary drastically from peak hour to off peak of the day exists, especially in urban area. Furthermore, the number of users may also increase but gradually in residential areas due to the growth in population. To evaluate how the NSSs under study cope with the variation of user density, an evaluation is carried out by varying the number of users in the HetNet cell. A scenario with $7 \mathrm{WiFi}$ APs, a medium WiFi density, is chosen and WiFi-LTE Coverage overlap is set to $100 \%$. The number of users is increased from 100 to 250 , in 50 user increments (Figure 14).

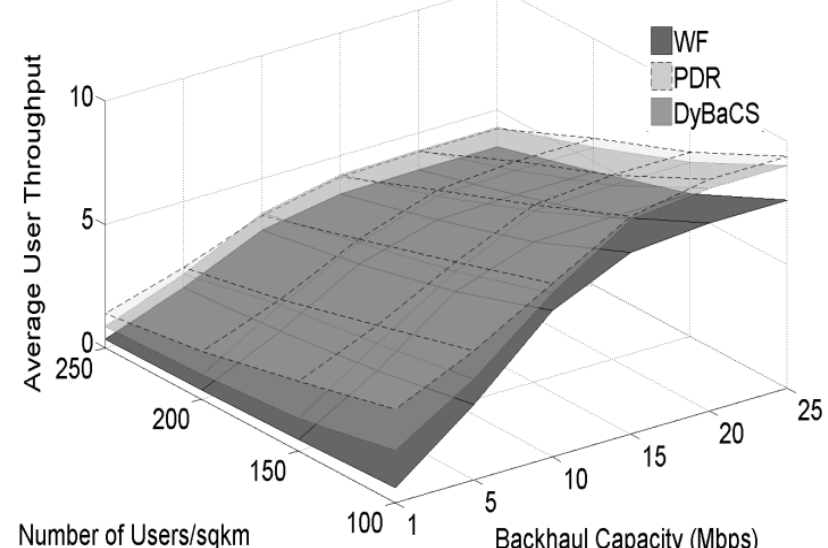

(a) Average User Throughput



(b) Fairness

Figure 14: NSSs performance as a function of Number of Users and Backhaul capacity. (7 WiFi APs, 100\% WiFi-LTE Coverage)

Figure 14 shows that PDR provides an advantage on average user throughput over DyBaCS when WiFi backhaul capacity values are low (i.e. $1 \mathrm{Mbps}$ to $5 \mathrm{Mbps}$ ) regardless of user density. However, when the WiFi backhaul capacity is increased, the difference in average user throughput between PDR and DyBaCS becomes negligible. At highest user density with 250 users per square kilometer, average user throughput of DyBaCS is greater than PDR when WiFi backhaul capacity exceeds $10 \mathrm{Mbps}$. WF remains the worst in providing capacity to users. DyBaCS remains the best scheme in terms of fairness for the entire range of user density and backhaul capacity.

Figure 14 implies that with increasing number of users, PDR is actually losing its advantage on average user throughput and fairness to DyBaCS and WF respectively, accentuated in Figure 15 which depicts NSSs performance for all three $\mathrm{WiFi}$ node densities considering the highest number of users (250 users). Figure 15 shows that with 250 users, PDR is the worst in terms of fairness despite previous results (refer to Figure 13 and Figure 14) showing that PDR is the second best on fairness. PDR's advantage on average user throughput is also sliced to a thin margin when both number of users and WiFi node density is high. For instance, in Figure 15, when number of WiFi nodes is 4, PDR is much superior in terms of average user throughput. 


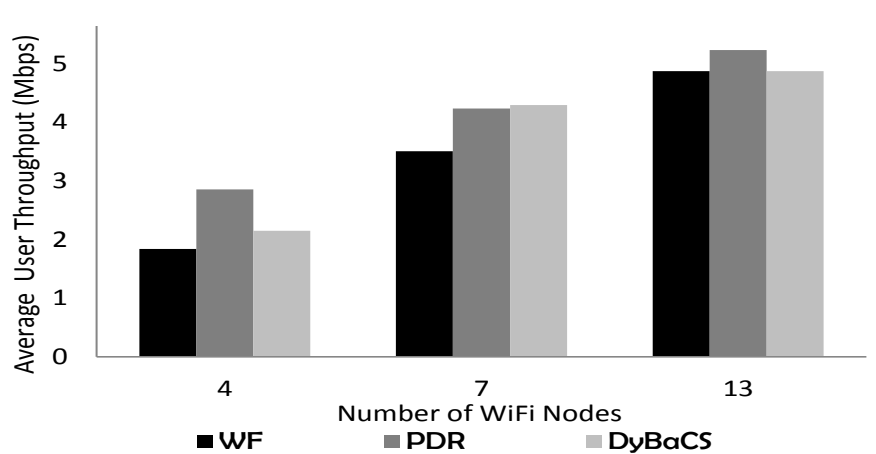

(a) Average User Throughput

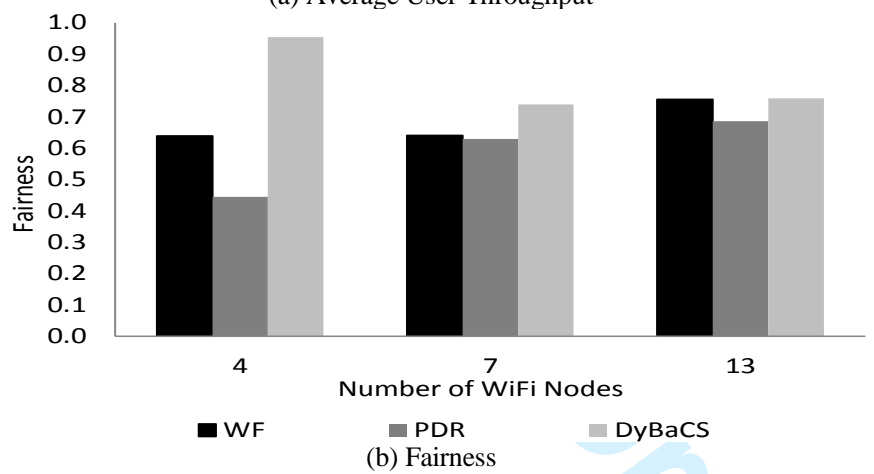

Figure 15: Performance comparison between NSSs as a function of total WiFi Nodes with 250 Users and fixed backhaul capacity of 20Mbps

However, at higher WiFi node density, PDR's advantage on average user throughput becomes insignificant.

As a general conclusion DyBaCS is good for fairness whilst maintaining the average user throughput, a clear advantage when the number of users is high with dense AP deployment, a typical urban scenario. It is adaptive to varying number of WiFi nodes and varying number of users, highly relevant in fast changing environments. PDR which is a form of greedy algorithm maximizes the average user throughput but at the expense of compromised fairness and its advantages in cell throughput diminish when both number of users and AP density are high. Although WF performs adequately compared to PDR when APs density, user density and backhaul capacity are high, it performs poorly otherwise and never outperforms DyBaCS in all scenarios under study.

\subsection{Performance on Non-uniform Backhaul Capacity}

In another scenario with 100 users, backhaul capacity of 4 APs covering the entire HetNet are non-uniformly assigned with AP1, AP2, AP3 and AP2 equal to $10 \mathrm{Mbps}, 0.5 \mathrm{Mbps}$, $20 \mathrm{Mbps}$ and $3 \mathrm{Mbps}$ respectively. Average user throughput is presented in Figure 16a, where PDR has the highest value (4.75Mbps) followed by DyBaCS (4.22Mbps) and WF (2.62Mbps). PDR is expected to offer higher average user throughput as it selects the network based on the highest physical data rate with the intention of maximizing the average user throughput. However, it degrades the fairness in terms of capacity distribution among the users highlighted in Figure 16b where the fairness index of PDR is only 0.59 compared to 0.86 by using DyBaCS. Although the average throughput of DyBaCS is $11 \%$ lower than PDR, but the traded off seems to be worthwhile as fairness is significantly improved by $45 \%$. WF only provides a 0.43 fairness index.



(a) Average User Throughput

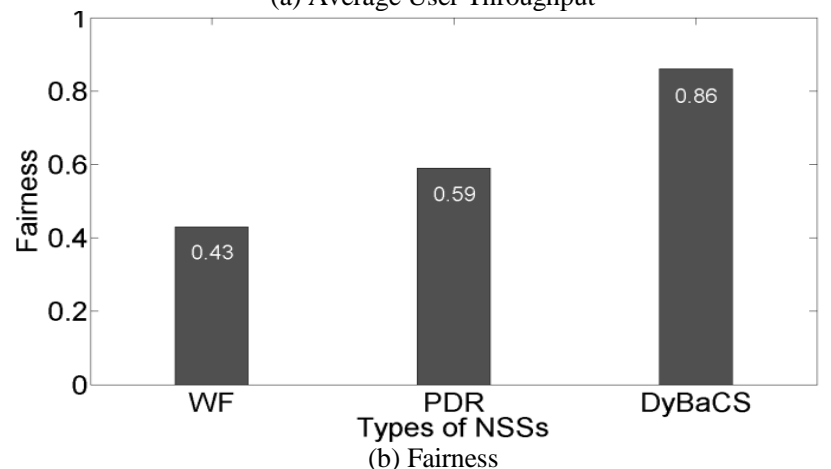

Figure 16: (a) Average user throughput and (b) Fairness on bandwidth sharing with non-uniform WiFi backhaul capacity. (4 Nodes, 100 users)



(a) Average User Throughput

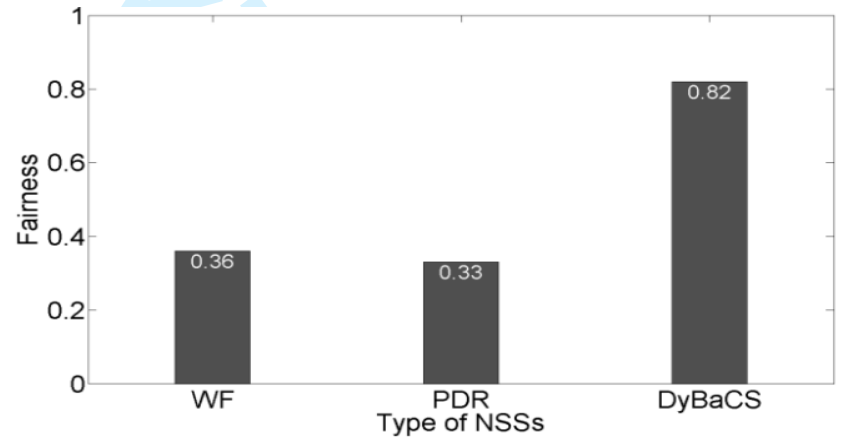

(b) Fairness

Figure 17: (a) Average user throughput and (b) Fairness of bandwidth sharing with non-uniform WiFi backhaul capacity. (4 Nodes, 250 users)

Using a similar scenario but by increasing the number of users to 250 , Figure $17 \mathrm{~b}$ shows that fairness index offered by PDR (at 0.33) is the poorest amongst all the NSSs under study although as shown in Figure 17a, PDR provides the highest 
average user throughput. DyBaCS on the other hand provides a good balance between average user throughput and fairness.

The results captured in Figure 16 and Figure 17 consistently indicate that DyBaCS, under non-uniform WiFi backhaul scenarios, provisions best fairness to users with minimal penalty in the average user throughput. DyBaCS provides the best performance on both average throughput and fairness when both user density and WiFi node density is high ( similar to uniform backhaul capacity case). Performance under non-uniform WiFi backhaul is in agreement with that of uniform backhaul evaluation detailed in Section 4. However, it is important to note that changes in user location and distribution of backhaul capacity may affect performance to a certain degree but the general trends remain.

\section{Conclusions}

A backhaul capacity sensitive network selection scheme referred to as DyBaCS within a wireless HetNet has been presented. The performance of DyBaCS and two commonly used NSSs has been evaluated as a function of WiFi backhaul capacity, WiFi-LTE coverage ratio, WiFi-LTE node ratio and user density. The performance of NSSs has also been compared under uniform and non-uniform WiFi backhaul capacity distributions. Results show that the proposed DyBaCS scheme provides the best fairness whilst preserving the average user throughput over all scenarios. DyBaCS is a highly scalable NSS as compared to PDR and WF in scenarios with varying number of users, WiFi nodes and WiFi backhaul capacity. Future work needs to consider more realistic proportional fairness capacity sharing schemes. The use of higher capacity LTE base stations and IEEE802.11n WiFi also needs to be studied. Besides, evaluation under different traffic types also needs to be addressed.

\section{Acknowledgment}

The work is supported by Ministry of Science, Technology and Innovation of Malaysia under the Science Fund project code 01-03-04-SF0011.

\section{References}

1. The LTE capacity shortfall: Why small cell backhaul is the answer, Aviat Networks, Retrieved 14th May 2013 from http:// www.aviatnetwork.com/

2. Small cell market status Feb 2013, Informa telecoms \& media, Retrieved 2nd October 2013 from http://www.smallcellforum.org/

3. Small cell operators face myriad operational and financial challenges, Infonetics Research, Retrieved 1st Oct 2013 from http://www.infonetics.com/

4. Mark Priscaro (14th May 2013), Telecom New Zealand and Ruckus Wireless Transform Traditional Phone Booths into Super Fast Wi-Fi Hotspots, Ruckus Wireless, Retrieved 16th May 2013 from http:// www.ruckuswireless.com/

5. Firetide Wireless Mesh Solutions for TELCOS: Access \& Small Cell Backhaul, https://www.winncom.com/manufacturer/firetide
6. Karam F W, Jensen T. Performance analysis of ranking for QoS handover algorithm for selection of access network in heterogeneous wireless networks. 21st International Conference onComputer Communications and Networks (ICCCN), 30 July-2 Aug 2012:1-6.

7. A Handa. Mobile Data Offload for $3 \mathrm{G}$ Networks: A White Paper. http://www.intellinettech.com/Media/PagePDF/Data\%20Offload.pdf, Oct. 2009.

8. J Nie, J Wen, Q Dong, Z Zhou. A seamless handoff in IEEE802.16a and IEEE802.11n hybrid networks, in proc. Int. Conf. Communi. Circuits Syst 2005, 1: 383-387.

9. J Roy, V Vaidehi, and S Srikanth. Always best-connected QoS integration model for the WLAN, WiMAX heterogeneous network. In IEEE First International Conference on Industrial and Information Systems, 8-11 Aug 2006: 361-366.

10. Y Bejerano, S J Han, and L E Li. Fairness and Load Balancing in Wireless LANs Using Association Control, IEEE/ACM Transactions on Networking 2007; 15(3):560573.

11. Judd G, Steenkiste P. Fixing 802. 11 access point selection. ACM SIGCOMM Computer Communication Review 2002; 32(3): 31-31.

12. D Kumar, E Altman, and J M Kelif. Globally optimal user-network association in an 802.11 WLAN and 3G UMTS hybrid cell. International Teletraffic Congress, Ottawa, Canada, June 17-21 2007: 1173-1187.

13. K Premkumar and A Kumar. Optimum Association of Mobile Wireless Devices with a WLAN-3G Access Network, Proc. IEEE Int'l Conf. Comm. (ICC '06), 11-15 June 2006: 2002-2008.

14. S. Singh, H. S. Dhillon, and J. G. Andrews. Offloading in heterogeneous networks: Modeling, analysis, and design insights, IEEE Trans. Wireless Communications 2013; 12(5): 2482-2497.

15. P Li , J Kong, S H Kim, H B Jung, X L Niu, D K Kim. Power allocation with max-min fairness for multicast in heterogeneous network, 14th International Conference on Advanced Communication Technology (ICACT), 19-22 Feb 2012: 502-505

16. P Xue, P Gong, J H Park, D Y Park, D K Kim. Radio resource management with proportional rate constraint in the heterogeneous networks, IEEE Transactions on Wireless Communications 2011; 11(3): 1066-1075.

17. Y Bejerano and S J Han. Cell Breathing Techniques for Load Balancing in Wireless LANs. IEEE Tranctions on Mobile Computing 2009; 8(6): 735-749.

18. H Velayos, V Aleo, and G Karlsson. Load Balancing in Overlapping Wireless LAN Cells, Proc. IEEE International Conference on Communications, 20-24 June 2004: 3833-3836.

19. Pei X, Jiang T, Qu D, Zhu G \& Liu J. Radio-resource management and access-control mechanism based on a novel economic model in heterogeneous wireless networks. IEEE Transactions on Vehicular Technology 2010, 59(6): 3047-3056.

20. Zhuo $\mathrm{X}$, Hua $\mathrm{S}$, Gao $\mathrm{W} \& \mathrm{Cao} \mathrm{G}$. An Incentive Framework for Cellular Traffic Offloading. IEEE Transactions on Mobile Computing 2014; 13(3): 541-555.

21. Yang Z, Yang Q, Fu F \& Kwak K S. A novel load balancing scheme in LTE and WiFi coexisted network for OFDMA system. International Conference on Wireless 
Communications \& Signal Processing (WCSP), 24-26 Oct 2013; 1-5.

22. F Bari and V C M Leung. Automated network selection in a heterogeneous wireless network environment. IEEE Network 2007; 21(1): 34-40.

23. $\mathrm{H} \mathrm{Hu}, \mathrm{W}$ Zhou, S Zhang, J Song. A Novel network selection algorithm in next generation heterogeneous network for modern service industry. IEEE Asia-Pacific Services Computing Conference, 9-12 Dec 2008: 12631268.

24. Galeana-Zapien H, Ferrus R. Design and Evaluation of a Backhaul-Aware Base Station Assignment Algorithm for OFDMA-Based Cellular Networks. IEEE Transactions on Wireless Communications 2010; 9(10): 3226-3237.

25. Pahlavan $\mathrm{K}_{\text {\& }}$ \& Levesque $\mathrm{A} \mathrm{H}$. Wirless Information Networks, $2^{\text {nd }}$ Edition, John Wiley \& Sons, 2005.

26. P Bender et al. CDMA/HDR: A Bandwidth Efficient High-speed Wireless Data Service for Nomadic User. IEEE Communication Magazine 2000; 38(7): 70-77.

27. Gerasimenko M, Moltchanov D, Florea R, Andreev S, Koucheryavy Y, Himayat N, Yeh S-p. \& Talwar S. Cooperative Radio Resource Management in Heterogeneous Cloud Radio Access Networks. IEEE Access 2015; 3: 397-406.

28. Yang $\mathrm{C}, \mathrm{Li} \mathrm{J}$ \& Anpalagan A. Cooperative bargaining game - theoretic methodology for $5 \mathrm{G}$ wireless heterogeneous networks. Transactions on Emerging Telecommunications Technologies 2014; 26(1): 70-81.

29. Bredel M \& Fidler M. A measurement study of bandwidth estimation in IEEE $802.11 \mathrm{~g}$ wireless LANs using the DCF. NETWORKING 2008 Ad Hoc and Sensor Networks, Wireless Networks, Next Generation Internet; 4928: 314325. Springer.
30. Yongmin Choi, Hyun Wook Ji, Jae-yoon Park, Hyun-chul Kim, Silvester J A. A $3 \mathrm{~W}$ network strategy for mobile data traffic offloading. IEEE Communications Magazine 2011; 49(10): 118-123.

31. Tranzeo Wireless Technologies Inc. Example Community Broadband Wireless Mesh Network Design, Version 1.1, 20June 2007.

32. NanoStation2, Ubiquiti Networks, www.ubnt.com/products/nano.php

33. A Ting, $\mathrm{D}$ Chieng, $\mathrm{K} \mathrm{H}$ Kwong, I Andonovic. Optimization of Heterogeneous Multi-radio Multi-hop Rural Wireless Network. IEEE $14^{\text {th }}$ International Conference on Communicaiton Technology 2012, ChengDu China, 9-11 Nov 2012: 1159-1165.

34. 3GPP, Evolved Universal Terrestrial Radio Access (EUTRA); Radio Frequency (RF) system scenarios (Release 10), 3GPP TR 36.942 V10.2.0 2010

35. ITU-R Report M.2135-1: Guidelines for evaluation of radio interface technologies for IMT-Advanced, December 2009.

36. M Petrova, P Mähönen, and J Riihijärvi. Connectivity analysis of clustered ad hoc and mesh networks, In Proceedings of IEEE GLOBECOM, Martinique 26-30 Nov 2007: 1139-1143.

37. R Jain, A Durresi, and G Babic. Throughput fairness index: an explanation, ATM Forum/99-0045, Feb. 1999. 


\section{Scalability Study of Backhaul Capacity Sensitive} Network Selection Scheme in LTE-WiFi HetNet

\author{
Alvin Ting, David Chieng, \\ Kae Hsiang Kwong \\ Wireless Communication, MIMOS \\ Berhad, K.L, Malaysia. \{kee.ting, \\ ht.chieng, kh.kwong\}@mimos.my
}

\author{
Ivan Andonovic \\ CIDCOM, Dept. EEE, University of \\ Strathclyde, Scotland, UK \\ i.andonovic@eee.strath.ac.uk
}

\author{
K. D. Wong \\ Daniel Wireless LLC \\ Palo Alto, CA, USA \\ dwong@danielwireless.com
}

\begin{abstract}
Wireless Heterogeneous Network (HetNet) with small cells presents a new backhauling challenge which differs from those of experienced by conventional macro-cells. In practice, the choice of backhaul technology for these small cells whether fiber, xDSL, point-to-point and point-to-multipoint wireless, or multi-hop/mesh networks, is often governed by availability and cost, and not by required capacity. Therefore, the resulting backhaul capacity of the small cells in HetNet is likely to be non-uniform due to the mixture of backhaul technologies adopted. In such an environment, a question then arises whether a network selection strategy that considers the small cells' backhaul capacity will improve the end users' usage experience. In this paper, a novel Dynamic Backhaul Capacity Sensitive (DyBaCS) network selection schemes (NSS) is proposed and compared with two commonly used network NSSs, namely WiFi First (WF) and Physical Data Rate (PDR) in an LTE-WiFi HetNet environment. The proposed scheme is evaluated in terms of average connection or user throughput ${ }^{1}$ and fairness among users. The effects of varying WiFi backhaul capacity (uniform and non-uniform distribution), WiFi-LTE coverage ratio, user density and WiFi access points (APs) density within the HetNet form the focus of this paper. Results show that the DyBaCS scheme generally provides superior fairness and user throughput performance across the range of backhaul capacity considered. Besides, DyBaCS is able to scale much better than WF and PDR across different user and WiFi densities.
\end{abstract}

Keywords - Hetrogeneous Network, Network Selection, LTE, WiFi, Traffic Offload, Backhaul Capacity

\section{Introduction}

The increasing pressure for mobile operators to offload data traffic from their $3 \mathrm{G}$, LTE or WiMAX networks to small cell networks [2] [3] indicates that future mobile broadband networks will largely be heterogeneous. This migration is further fueled by the availability of multi-RAT (Radio Access Technology) which allows user devices to connect to different wireless networks such as 3G, LTE, WiMAX, WiFi either one

${ }^{1}$ Capacity and throughput are used interchangeably throughout article with the detail definition in section 2.1

* A shorter version of this article is presented in [1]. This article includes substantial revisions, enhancements and extensions to [1]. at a time or simultaneously. Deployment of small cells such as WiFi raises new backhaul challenges for operators. There are only two broad choices as far as backhaul is concerned, wireline and wireless backhaul. Due to intensive engineering work required, high cost and regulatory barriers, fixed line solutions such as fiber, cable, copper or xDSL are not ubiquitously available. Further, a relatively large number of WiFi hotspots may prove too costly for operators to backhaul with wired options. In such situations, the operator may instead increasingly rely on point-to-point and point-to-multipoint wireless solutions. However in most scenarios, a mixture of backhaul technologies is expected, as operators will adopt the most suitable backhaul solution for small cells considering the cost and availability [4] (Figure 1). With the maturity of multihop mesh networks, it may also serve as a potential candidate [5] [6].

Currently, WiFi APs are typically backhauled through different types of technologies which offer throughput capacity ranging from several mega bit per second (Mbps) to tens of Mbps [5]. Backhauling APs using different technologies within the HetNet leads to non-uniform AP capacity distribution. The most widely deployed IEEE802.11n WiFi technology which provisions a peak physical data rate of $600 \mathrm{Mbps}$. Due to that most existing fixed backhaul services are not able to offer sufficient capacity for these WiFi APs to realize their full potential. Therefore consideration of WiFi backhaul capacity is inevitable during traffic offload decision making.

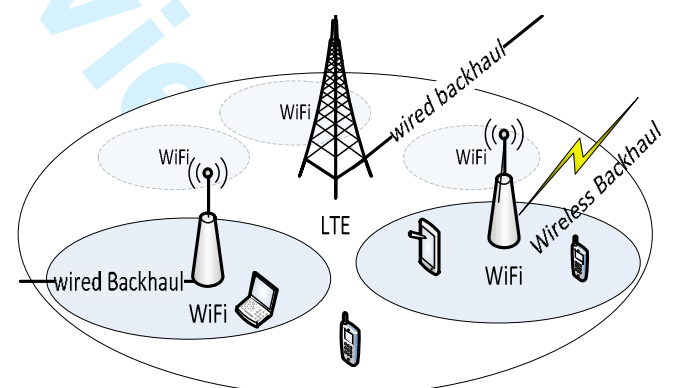

Figure 1: LTE-WiFi Heterogeneous Network with different backhaul options.

\subsection{Related Work}

To reduce data traffic pressure over mobile networks, alternative networks such as WiFi are preferred by mobile operators whenever possible [7]. As report in [8], most WiFi-enabled smartphones are configured with WiFi First 
network selection scheme by default to give WiFi higher priority over the cellular interface for data transmissions. Network selection strategies for WiMAX-WiFi network which is predominantly driven by data rate are reported in [9] and [10]. This kind of network selection strategies lead to poor user experiences [11] and causes unbalanced load distribution amongst access networks [12].

Several studies [13-15] have proposed the use of Network Selection Schemes (NSSs) to optimize network performance. The work by [13] considers a globally optimal user-network association scheme in a WLAN-UMTS hybrid cell. In [14], a heuristic greedy search algorithm that maximizes total user throughput in a heterogeneous wireless access network comprising WiFi APs and $3 \mathrm{G}$ BSs is proposed. Both these studies adopt a simplified WLAN model by assuming only one transmission rate. Poisson point process (PPP) theory and stochastic geometry method are used in [15] to model traffic offloading in a multi RAT heterogeneous wireless network. The authors propose a method to determine the optimum percentage of the traffic that needs be offloaded in order to maximize network coverage while meeting user requirements. In that work however, no network selection algorithm is proposed.

Fairness performance is considered in [16-19] where [16] focuses on ensuring max-min fairness for multicast in Orthogonal Frequency Division Multiple Access (OFDMA)based wireless heterogeneous networks. A proportional user rate based radio resource management strategy is investigated in [17] on LTE-WiFi HetNet, where a suboptimal network selection algorithm is introduced to improve the minimum normalized user rate and fairness. Bejerano et al. [18] proposes changes to the transmission power of AP beacon messages in order to minimize the load in congested APs and produces an optimal max-min load balancing strategy. A load balancing scheme for overlapping wireless LAN cells is reported in [19]. However, the work aims to balance the throughput of APs and not the fairness amongst users.

NSSs that consider QoS are reported in [7] [23] [24]. Network selection based on multiple parameters such as cost, bandwidth and QoS parameters including packet loss, jitter and delay is reported in [23]. However, the study focuses only on general heterogeneous networking and no specific type of technology is simulated. Similarly, the work in [7] and [24] investigate access network selection for optimal service delivery and QoS to users respectively. However the overall HetNet performance and fairness is not considered.

Load balancing in Cellular and WLAN HetNet is reported in [20] [21] and [22]. In [20], a joint access-control strategy is designed for sharing of the radio resource and load balancing between CDMA Cellular Network and WLANs by considering user preferences. Bandwidth allocation is optimized by maximizing the aggregated social welfare of the WLANs under interference-constraint environment. However, the method used to balance the load amongst mobile nodes is not disclosed. The work in [21] looks into the trade-off between the amount of traffic being offloaded and users' satisfaction. An incentive framework is proposed to motivate users to use delay tolerance WiFi networks for traffic offloading. Yang et al. in [22] proposes a load balancing scheme that aims to balance the network load between the LTE network and WiFi hotspots considering access pattern of UEs.

Bejerano et al. [11] considers backhaul capacity during AP selection, while [25] proposes a backhaul-aware base station (BS) selection algorithm. Although backhaul-aware network selection scheme have been explored in these works, their scope is limited to homogeneous wireless networks.

In this paper, a Dynamic Backhaul Capacity Sensitive (DyBaCS) NSS is proposed for LTE-WiFi HetNet. This scheme not only considers access link throughput but also available backhaul capacity in order to preserve fairness amongst users. The proposed scheme is compared with two commonly used NSSs namely WiFi First (WF) and Physical Data Rate (PDR). This paper investigates fairness and average user throughput performances across WiFi backhaul capacity ranging from $1 \mathrm{Mbps}$ to $25 \mathrm{Mbps}$.

The rest of the paper is organized as follows. Section 2 describes the proposed DyBaCS network selection algorithm as well as WF and PDR schemes. Section 3 details the simulation approach, simulation parameters and assumptions made. Results are discussed in Section 4 followed by conclusions in Section 5 .

\section{Existing and Proposed Network Selection Schemes}

This section describes the simulation approach simulation parameters and assumptions used in the study. The notations used are listed in TABLE I.

\begin{tabular}{|c|c|}
\hline Notation & Description \\
\hline$N_{\text {cluster }}$ & number of clusters for user distribution \\
\hline$\sigma_{x}, \sigma_{y}$ & width and height of user distribution clusters \\
\hline$N_{\text {user }}$ & number of users in a cluster \\
\hline SINR & signal to interference and noise ratio \\
\hline SNR & signal to noise ratio \\
\hline$p_{u}$ & WiFi receive power by user $u$ \\
\hline$p_{I}$ & WiFi interference power \\
\hline$N F$ & noise floor \\
\hline$C_{A v}$ & average user throughput \\
\hline$R$ & WiFi downlink physical transmission rate to users \\
\hline$\varphi$ & link throughput efficiency measured at the IP layer \\
\hline$T$ & $\begin{array}{l}\text { maximum downlink throughput achievable by a LTE } \\
\text { user }\end{array}$ \\
\hline$P L$ & path loss in $\mathrm{dB}$ \\
\hline$d$ & distance in meters \\
\hline NLOS & Non-line-of-sight \\
\hline OF & overbooking factor \\
\hline EIRP & effective isotropic radiated power \\
\hline WF & WiFi First network selection scheme \\
\hline DyBaCS & $\begin{array}{l}\text { Dynamic Backhaul Capacity Sensitive network selection } \\
\text { scheme }\end{array}$ \\
\hline PDR & Physical Data Rate Based network selection scheme \\
\hline MAC & medium access control \\
\hline UE & user equipment \\
\hline$C_{\text {sys }}^{i}$ & system throughput of AP $i$ \\
\hline$C_{s y s}^{L T E}$ & LTE system throughput within the HetNet \\
\hline$N_{u s e r}^{i}$ & total number of users in $\mathrm{AP} i$ \\
\hline
\end{tabular}




\begin{tabular}{|c|l|}
\hline$N_{\text {user }}^{L T E}$ & total number of users in a LTE cell \\
\hline$N_{\text {user }}^{\text {HetNet }}$ & total number of users in HetNet \\
\hline$C_{\text {link,u }}^{i}$ & access link throughput from AP $i$ to user $u$ \\
\hline$C_{\text {link,u }}^{L T E}$ & access link throughput from LTE BS to user $u$ \\
\hline$C_{b h}^{i}$ & backhaul capacity of AP $i$ in Mbps \\
\hline$C_{A v, l t d}^{i}$ & $\begin{array}{l}\text { average AP } i \text { users' throughput with limited backhaul } \\
\text { capacity }\end{array}$ \\
\hline$C_{A v}^{i}$ & $\begin{array}{l}\text { average AP } i \text { users' throughput with sufficient backhaul } \\
\text { capacity }\end{array}$ \\
\hline$C_{e f f}$ & effective user throughput \\
\hline$C_{n}^{i}$ & estimated throughput if user $n$ is connected to AP $i$ \\
\hline$C_{n}^{L T E}$ & estimated throughput if user $n$ is connected to LTE BS \\
\hline$\Omega$ & $\begin{array}{l}\text { list of higher speed connections (amongst WiFi and LTE } \\
\text { interfaces for a user) for all users in throughput }\end{array}$ \\
\hline$A$ & set that contains all users in the HetNet \\
\hline
\end{tabular}

\subsection{Network Capacity Model}

\subsubsection{Single WiFi AP with Max-min Fairness}

In a multi-rate WiFi network, users with slower link speed tend to occupy more spectrum resource (in terms of transmit time) than higher speed users for the same amount of data sent. Such a bandwidth sharing scenario amongst all users within a single AP coverage has been modelled.

Suppose a total of $N_{\text {user }}$ users are connected to an AP and each is supported by a data rate, $R_{u}$ in accordance to their $S I N R$ level. Thus, user $u$ receives transmission slots at rate $R_{u}$ bps, where $u=1,2 . . N_{\text {user }}$. Assuming the channel access probability is $\mathcal{P}_{\mathrm{u}}$ and it exhibits the effect of slots assignment, which is inversely proportional to user data rate $R_{u}$. Let $S_{u}$ be the number of slots allocated to user $u$, where $S_{u}=k / R_{u}$, with $k$ being an arbitrary constant which will be cancelled off eventually. In this case, if user packet size is assumed to be the same, the AP throughput at the physical layer can be calculated as [26] [27]:

$$
C_{\text {sys }}^{\text {Phy }}=\frac{\sum_{u=1}^{N_{u s e r}} \mathcal{P}_{u} R_{u} S_{u}}{\sum_{u=1}^{N_{u s e r}} \mathcal{P}_{u} S_{u}}
$$

Substituting $S_{u}=\frac{k}{R_{u}}$ into Eqn (1);

$$
C_{s y s}^{\text {Phy }}=\frac{\sum_{u=1}^{N_{u s e r}} \mathcal{P}_{u} R_{u}\left(\frac{k}{R}\right)}{\sum_{u=1}^{N_{u s e r}} \mathcal{P}_{u}\left(\frac{k}{R_{u}}\right)}=\frac{k \cdot \sum_{u=1}^{N_{u s e r}} \mathcal{P}_{u}}{k \cdot \sum_{u=1}^{N_{u s e r}} \frac{\mathcal{P}_{u}}{R} u}=\frac{\sum_{u=1}^{N_{u s e r}} \mathcal{P}_{u}}{\sum_{u=1}^{N_{u s e r}} \frac{\mathcal{P}_{u}}{R} u}
$$

Since the sum of $\mathcal{P}_{\mathbf{u}}$ by all user is $\sum_{u=1}^{N_{u s e r}} \mathcal{P}_{u}=1$, Eqn (2) can be further simplified to:

$$
C_{\text {sys }}^{\text {Phy }}=\frac{1}{\sum_{u=1}^{N_{u s e r}} \frac{\mathcal{P}_{u}}{R} u}
$$

The average physical layer throughput per user $C_{A v}^{P h y}$ is calculated by dividing Eqn (3) with the total number of users $N_{\text {user }}$ Since fair resource sharing is assumed, which in turn implies that the packet sending frequency of every user is the same, the $\mathcal{P}_{u}$ of a user can also be written as $\frac{1}{N_{u s e r}}$ :

$$
C_{A v}^{P h y}=\left(\frac{1}{N_{u s e r}}\right) \times\left(\frac{1}{\sum_{u=1}^{N_{u s e r} \frac{1}{N_{u s e r}}}}\right)=\frac{1}{\sum_{u=1}^{N_{u s e r}} \frac{1}{R_{u}}}
$$

The model facilitates max-min fairness [28] [29] behavior in a multi-rate system where all users are allocated an equal amount bandwidth resource. This characteristic is representative of WLAN Distributed Coordinated Function (DCF) [30] where all users are offered a fair medium access opportunity.

Example 1: Assume two users are connected to an AP with supported data rate of $R_{1}=54 \mathrm{Mbps}$ and $R_{2}=6 \mathrm{Mbps}$ respectively.

The number of time slots assigned is inversely proportional to data rate hence $S_{1}=\frac{k}{R_{1}}=\frac{k}{54}$ and $S_{2}=\frac{k}{R_{2}}=\frac{k}{6}$, where $k$ is an arbitrary constant. The channel access probability of user 1 and user 2 are represented by $\mathcal{P}_{1}$ and $\mathcal{P}_{2}$. Since both have a fair chance to transmit to the AP or vice versa, $\mathcal{P}_{1}=\mathcal{P}_{2}=\frac{1}{2}$. With both users taking turn to transmit packets and if their packet sizes are the same, the time taken by each user to transmit a packet can be represented by Figure 2 .



Figure 2: Time slots required by user1 and user2 considering fair throughput

From Figure 2, User 2 takes more time than User 1 in order to maintain throughput fairness. In other words, User 1 is being penalized in terms of time slot fairness, which is the characteristic of WiFi DCF system. The average throughput for both users can be calculated using Eqn (5) as follows:

$$
C_{A V}^{P h y}=\frac{S_{1}}{S_{1}+S_{2}} \times R_{1}=\frac{S_{2}}{S_{1}+S_{2}} \times R_{2}
$$

By substituting the values of $S_{1}, S_{2}, R_{1}$ and $R_{2}$ into Eqn (5), the average throughput for both users can be calculated as 5.4 Mbps.

Similarly, within a single WiFi network, the average IP layer throughput per user $C_{A v}$ (Eqn (6)) is calculated by multiplying the physical data rate $R_{u}$ in Eqn (4) by a corresponding throughput efficiency factor $\varphi_{u}$ given in TABLE V:

$$
C_{A v}=\frac{1}{\sum_{u=1}^{N u s e r} \frac{1}{R u \times \varphi_{u}}}
$$

The accuracy of the model (implementation of Eqn (6)) is verified together with the IEEE802.11g WiFi models (in 
section 3.1) using the QualNet event-driven simulator (Version 5.2) and the results are found to be very similar.

\subsubsection{LTE with Max-Min Fairness}

In LTE, resource is assigned to users in forms of Physical Resource Blocks (PRB) consisting of many Resource Elements. The same max-min fair bandwidth sharing approach is adopted where in the LTE case, more PRBs are assigned to users experiencing worse channel behavior to ensure fairness. This assumption is reasonable since the LTE Medium Access Control (MAC) scheduler has not been fully defined in the standards. In order to simplify the study, resource element allocations in LTE are approximated as time fraction allocation in a TDMA manner as modeled in [17] and the average user throughput is defined by Eqn (7), a straightforward modification from Eqn (6):

$$
C_{A v}=\frac{1}{\sum_{u=1}^{N_{u s e r}^{L T E}} \frac{1}{T_{u}}}
$$

where $T_{u}$ is maximum link throughput achievable by LTE user $u$ and $N_{\text {user }}^{L T E}$ is the total number of LTE users.

\subsection{Network Selection Schemes}

In a heterogeneous wireless network comprising two or more technologies, user throughput fairness needs to be considered in two aspects, intra-network fairness and internetwork fairness. Intra-network fairness can be provided by AP or LTE BS to its associated users as the task is confined locally. With max-min fair capacity sharing of WiFi and LTE network (Eqn (6) and Eqn (7)), fairness is achievable amongst users under the same AP or BS. However, fair capacity sharing across the entire HetNet is not guaranteed and is strongly dependent on the implemented NSSs. The proposed DyBaCS is compared with two commonly used NSSs namely WiFi First (WF) and Physical Data Rate (PDR), the characteristics of which are as follows:

\subsubsection{WiFi First (WF)}

WiFi First (WF) connects a user to an AP whenever WiFi coverage is available. In other words, a user will never connect to a LTE network when there is WiFi coverage. This approach is adopted by most smart phones' and tablets' connection manager by default as the preferred data connection mode. In addition, this mode is also promoted by some mobile operators as a means to offload traffic from mobile networks [31] [8].

\subsubsection{Physical Data Rate (PDR)}

PDR chooses the network based on the Physical data rate of the RAT available to the user [9] [10]. Here the physical data rates provided by LTE and $\mathrm{WiFi}$ are compared and the RAT with higher physical data rate is chosen. Since PDR tends to assign more bandwidth to the users with better link capacity, it compromises user fairness, though the overall throughput performance might be good.

\subsubsection{Proposed Dynamic Backhaul Capacity Sensitive (DyBaCS) scheme}

DyBaCS is mainly proposed to preserve fairness while maximizing HetNet throughput. Thus DyBaCS throughput performance may not outperform PDR (which is optimum in maintaining HetNet throughput), but it is very close to PDR in most cases (Section 4). In the study, a single LTE network is assumed with $I(I \geq 1)$ denoting the total number of $\mathrm{WiFi}$ APs in the HetNet and i denoting the index of the network, i.e. network $i=1 \leq i \leq I$ is the $i$-th WiFi network, while, $u=1 \leq u \leq N_{\text {user }}^{\text {HetNet }}$ represents the $u$-th user in the HetNet and $U$ is the total user in entire HetNet. Total number of users in AP $i$ and LTE network is represented by $U^{i}$ and $U^{L T E}$ respectively.

Before explaining the DyBaCS algorithm in detail, Algorithm 1; the User Throughput Estimation Flow (UTEF) algorithm, which enables the estimation of $\mathrm{WiFi}$ user throughputs $C_{e f f, u}^{i}$ and LTE user throughputs $C_{e f f, u}^{L T E}$ is first presented as follows:

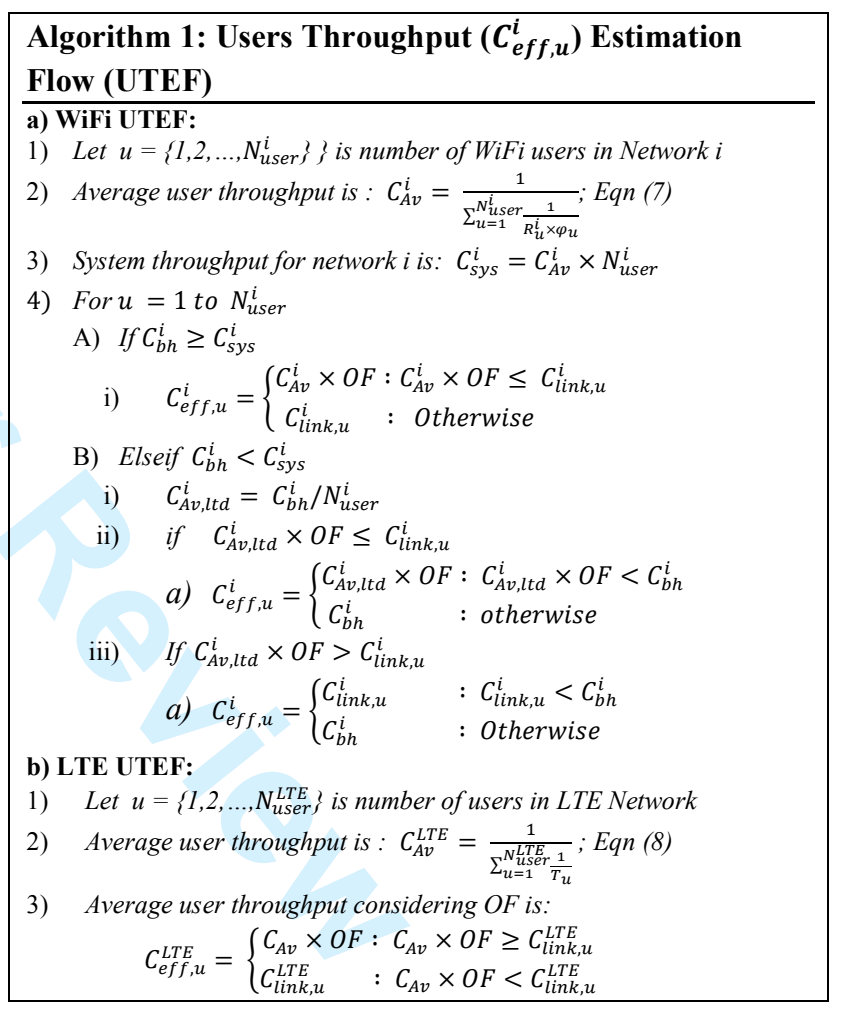

For WiFi UTEF (Algorithm 1), the calculation of effective WiFi user throughput $C_{e f f, u}^{i}$ is based on user access link throughput $C_{\text {link,u }}^{i}$, AP backhaul capacity $C_{b h}^{i}$ and Overbooking Factor (OF). Link throughput $C_{\text {link,u }}^{i}$ (or equivalent to $R_{u}^{i} \times \varphi_{u}$ in Eqn (6) and $\varphi_{u}$ is listed in TABLE V) of user $u$ is predominately affected by the distance from the $\mathrm{AP}$, channel quality and other channel conditions such as interference. Backhaul capacity $C_{b h}^{i}$ is defined as the actual available backhaul capacity to $\mathrm{AP} i$, whether it is wired or wireless backhaul. Effective user throughput is limited by both 
factors. In practice, OF is normally considered as not all the users access the channel at the same time [32]; by including OF the perceived throughput from the users point of view can be calculated.

The calculation of effective user throughput in WiFi UETF can be explained in detail with the aid of the scenario shown in Figure 3, where 4 users are connected to AP $i$. User data rate $R_{u}^{i}$ and link throughput $C_{\text {link,u }}^{i}$ are listed in TABLE II.

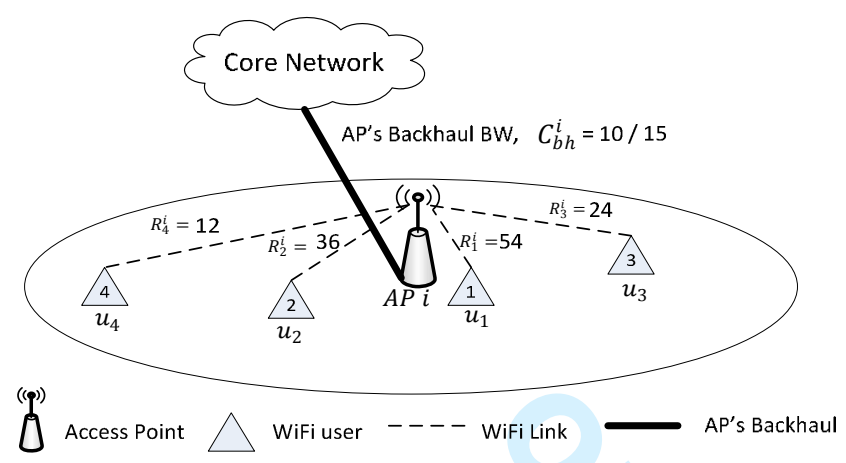

Figure 3: AP with 4 users under its coverage

\begin{tabular}{ccc}
\multicolumn{2}{c}{ TABLE II : USER DATA RATE AND LINK THROUGHPUT IN Figure 3 } \\
\hline User, u & $\boldsymbol{R}_{\boldsymbol{u}}^{\boldsymbol{i}}$ & $\boldsymbol{C}_{\boldsymbol{l i n} \boldsymbol{k}, \boldsymbol{u}}^{\boldsymbol{i}}=\boldsymbol{R}_{\boldsymbol{u}}^{\boldsymbol{i}} \times \boldsymbol{\varphi}_{\boldsymbol{u}}$ \\
\hline 1 & 54 & $54 \times 0.32=17.28$ \\
2 & 36 & $36 \times 0.41=14.76$ \\
3 & 24 & $24 \times 0.49=11.76$ \\
4 & 12 & $12 \times 0.61=7.32$ \\
\hline
\end{tabular}

Step 1 and Step 2 in Algorithm 1 estimate the average WiFi user throughput using Eqn (6) and the average user throughput can be calculated as:

$$
\begin{aligned}
C_{A v}^{i}= & \frac{1}{\sum_{u=1}^{N_{u s e r}^{i}} \frac{1}{R_{u}^{i} \times \varphi_{u}}}=\frac{1}{\frac{1}{R_{1}^{i} \times \varphi_{1}}+\frac{1}{R_{2}^{i} \times \varphi_{2}}+\frac{1}{R_{3}^{i} \times \varphi_{3}}+\frac{1}{R_{4}^{i} \times \varphi_{4}}} \\
= & \frac{1}{\frac{1}{54 \times 0.32}+\frac{1}{36 \times 0.41}+\frac{1}{24 \times 0.49}+\frac{1}{12 \times 0.61}} \\
& =2.88 \mathrm{M}
\end{aligned}
$$

Multiplying the average user throughput $C_{A v}^{i}$ by the total number of users $N_{u s e r}^{i}$, as in Step 3, yields the total system throughput $C_{s y s}^{i}$ of $\mathrm{AP} i$ :

$$
C_{s y s}^{i}=C_{A v}^{i} \times N_{u s e r}^{i}
$$

In the scenario, $C_{s y s}^{i}=2.88 \times 4=11.52 \mathrm{Mbps}$. As in Eqn (6), $C_{\text {sys }}^{i}$ is affected by link speed $R_{u}$ of all users in network $i$; therefore it is highly dependent on users distribution. $C_{s y s}^{i}$ represents the required backhaul capacity for AP $i$ to realise its full access capacity. If the backhaul capacity is sufficient to support the maximum capacity from the AP to clients such that $C_{b h}^{i} \geq C_{s y s}^{i}$, then all users will enjoy a throughput of $C_{A v}^{i}$. However, if the backhaul capacity $C_{b h}^{i}$ is the bottleneck, $C_{b h}^{i} \leq C_{s y s}^{i}$, the available backhaul capacity $C_{b h}^{i}$ is shared evenly to all users $N_{u s e r}^{i}$ and average user throughput in such scenario is limited to a value $C_{A v, l t d}^{i}$ (Eqn (10)) which is less than $C_{A v}^{i}$.

$$
C_{A v, l t d}^{i}=C_{b h}^{i} / N_{u s e r}^{i}
$$

It is important to note that users will not secure a throughput higher than $C_{A v}^{i}$ even at backhaul capacities greater than $C_{\text {sys }}^{i}$ due to the restriction imposed by the WiFi physical and MAC layer capability. Under the assumption of simultaneous channel access, $C_{A v}^{i}$ or $C_{A v, l t d}^{i}$ represents the average user throughput for both sufficient and limited backhaul capacity cases.

In Figure 3 , assuming that backhaul capacity $C_{b h}^{i}=10$ is less than $C_{\text {sys }}^{i}=11.52$, the actual average throughput for the users is limited to $C_{A v, l t d}^{i}=\frac{C_{b h}^{i}}{N_{u s e r}^{i}}=\frac{10}{4}=2.5 \mathrm{Mbps}$. Otherwise, each user will enjoy $C_{A v}^{i}=2.88 \mathrm{Mbps}$ if $C_{b h}^{i} \geq$ $C_{\text {sys }}^{i}$.

In order to simplify the explanation of Algorithm 1, an OF $=5$ is adopted for scenario depicted in Figure 3. Suppose $C_{b h}^{i}=$ $15 \mathrm{Mbps}$ where $C_{b h}^{i} \geq C_{s y s}^{i}$, the effective average throughput $C_{e f f, u}^{i}$ of user $u$ is only limited by its link throughput $C_{\text {link, } u}^{i}$. Step 4A (i) places a constraint to ensure that the value of $C_{A v}^{i} \times O F$ does not exceed $C_{\text {link } u}^{i}$. TABLE III shows that effective user throughput $C_{\text {eff }, u}^{i}$ is the minimum value between $C_{A v}^{i} \times O F$ and $C_{l i n k, u}^{i}$. Note that the effective average throughput for User 3 and User 4 is limited to their link throughput which is $11.76 \mathrm{Mbps}$ and $7.32 \mathrm{Mbps}$ respectively.

TABLE III: EFFECTIVE USER THEROUGHPUT $C_{e f f, u}^{i}$

\begin{tabular}{cccccc}
\multicolumn{6}{c}{ UNDER CONDITION $C_{\boldsymbol{b h}}^{i} \geq C_{\boldsymbol{s y s}}^{i}$} \\
\hline User, $\boldsymbol{u}$ & $\boldsymbol{C}_{\boldsymbol{A v}}^{\boldsymbol{i}}$ & $\boldsymbol{C}_{\boldsymbol{b h}}^{\boldsymbol{i}}$ & $\boldsymbol{C}_{\text {link } \boldsymbol{u} \boldsymbol{u}}$ & $\boldsymbol{C}_{\boldsymbol{A v}}^{\boldsymbol{i}} \times \boldsymbol{O F}$ & $\boldsymbol{C}_{\boldsymbol{e f f , u}}^{\boldsymbol{i}}$ \\
\hline 1 & 2.88 & 15 & 17.28 & 14.40 & 14.40 \\
2 & 2.88 & 15 & 14.76 & 14.40 & 14.40 \\
3 & 2.88 & 15 & 11.76 & 14.40 & 11.76 \\
4 & 2.88 & 15 & 7.32 & 14.40 & 7.32 \\
\hline
\end{tabular}

Likewise, with limited backhaul capacity, Step 4B (ii) and Step 4B (iii) ensure that $C_{A v, l t d}^{i} \times O F$ is bound by both $C_{\text {link, } u}^{i}$ and $C_{b h}^{i}$, whichever is smaller. The result is shown in TABLE IV.

TABLE IV : EFFECTIVE USER THROUGHPUT $C_{e f f, u}^{i}$

\begin{tabular}{cccccc}
\multicolumn{6}{c}{ UNDER CONDITION $C_{\boldsymbol{b h}}^{i} \leq C_{\boldsymbol{s y s} .}^{i}$} \\
\hline User, $\boldsymbol{u}$ & $\boldsymbol{C}_{\boldsymbol{A} \boldsymbol{v}}^{\boldsymbol{i}}$ & $\boldsymbol{C}_{\boldsymbol{b h}}^{\boldsymbol{i}}$ & $\boldsymbol{C}_{\text {link } \boldsymbol{u} \boldsymbol{u}}$ & $\boldsymbol{C}_{\boldsymbol{A v} \boldsymbol{v}}^{\boldsymbol{i}} \times \boldsymbol{O F}$ & $\boldsymbol{C}_{\boldsymbol{e f f}, \boldsymbol{u}}^{\boldsymbol{i}}$ \\
\hline 1 & 2.88 & 10 & 17.28 & 12.5 & 10 \\
2 & 2.88 & 10 & 14.76 & 12.5 & 10 \\
3 & 2.88 & 10 & 11.76 & 12.5 & 10 \\
4 & 2.88 & 10 & 7.32 & 12.5 & 7.32 \\
\hline
\end{tabular}


In short, Step 4 imposes logical considerations to the determination of the effective user average throughput $C_{e f f, u}^{i}$ taking into consideration backhaul capacity, user link speed and OF. Similarly, the LTE user throughput can also be estimated using the LTE UTEF approach. In this paper, LTE backhaul capacity is always assumed to be sufficient. Therefore in Step 3, the only constraint for user throughput in the LTE network is the access link throughput $C_{\text {link, }}^{L T E}$.

The DyBaCS NSS (Algorithm 2) assumes that initially, there is no user connected to the HetNet. Users are admitted to the HetNet one by one and their servicing network is determined by the NSS.

Step1 initializes variables $\Omega, A, N_{\text {user }}^{i}$ and $N_{\text {user }}^{L T E}$, where $\Omega$ is a list holding the higher speed connections between $\mathrm{WiFi}$ and LTE interfaces to users, $A$ is the set containing all users in the HetNet, $N_{u s e r}^{i}$ is the list of users connected to the WiFi AP $i$ and $N_{\text {user }}^{L T E}$ is the list of users connected to the LTE network.

In Step 2, the access link with the highest throughput between user $u$ to $\operatorname{LTE}\left(C_{\text {link }, u}^{L T E}\right)$ or WiFi $\left(C_{\text {link }, u}^{i}\right)$ is chosen and placed into $\Omega, \Omega_{u}$ represents the throughput value for user $u$. All users are able to connect to at least the LTE network while access to WiFi depends on availability of WiFi coverage. Hence $\beta_{u}^{i}=\{0,1\}$ denotes the connectivity of user $u$ to $\mathrm{AP} i, \beta_{u}^{i}=$ ' 1 ' means connection is possible, ' 0 ' means the opposite. Since multi-homing is not considered, every user is only allowed to connect to one network at a time. Step 3 assigns all users with no WiFi access to the LTE network and excludes those users from set $A$. The network selection parameter $\alpha_{u}^{i} \in\{0,1\}$ indicates the choice of user $u ; \alpha_{u}^{i}={ }^{\prime} 1$ ', indicates that user $u$ is connected to network $i$ and value ' 0 ', means the opposite.

The remaining users within set $A$ which have not been assigned to any network are subjected to network selection; they can either assigned to WiFi or LTE. Network selection for those users is addressed in Step 4 in order to optimize the network performance. Step 4 (a) ensures the user with the highest link throughput within set $A$ (referred to as user $n$ ) is considered first.

In Step 4 (b) and Step 4 (c), the achievable throughput of user $n$ (considering access link speed, AP backhaul capacity and OF) on WiFi AP $i$ and LTE is estimated using WiFi UTEF and LTE UTEF and represented by $C_{e f f, n}^{i}$ and $C_{e f f, n}^{L T E}$ respectively. The throughput estimation is executed assuming that user $n$ is added to the corresponding WiFi or LTE network. Therefore, during the throughput estimation, the total number of users connected to AP $i$ is assumed to be user $n$ plus the total number of existing users $N_{u s e r}^{i}$ written as $N_{u s e r}^{i} \cup\{n\}$ and similarly the total number of users in the LTE network is $N_{\text {user }}^{L T E} \cup\{n\}$. The achievable throughput for user $n$ on the WiFi and LTE network is then assigned to $C_{n}^{i}$ and $C_{n}^{L T E}$ respectively.

User $n$ is subsequently assigned to the network that offers the highest throughput and the corresponding $\alpha_{n}^{L T E}$ or $\alpha_{n}^{i}$ value is set according to Step 4 (d). The total number of users connected to their respective $i$-th WiFi network $N_{\text {user }}^{i}$ or LTE network $N_{\text {user }}^{L T E}$ is then updated.

Finally set $A$ is updated by excluding user $n$ in Step 4(e) and the Step 4 processes are repeated until all users are serviced.

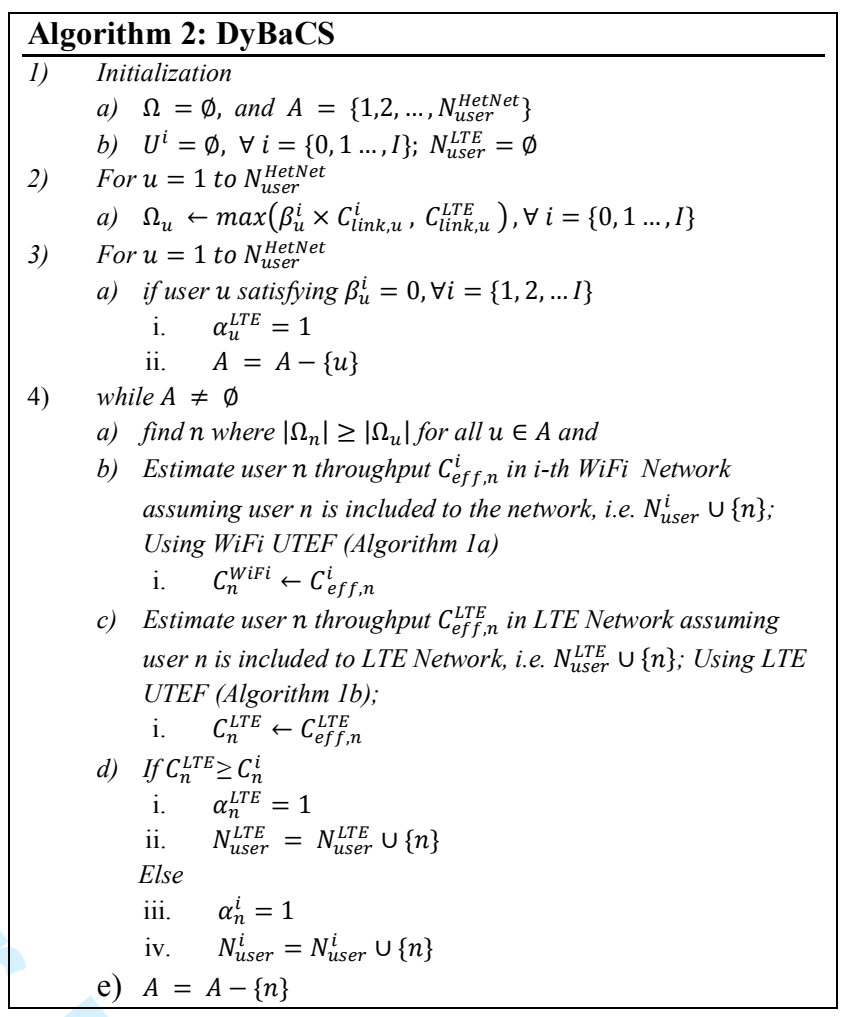

\section{Parameters, Assumptions and Simulation Approach}

The section describes the simulation approach, simulation parameters and assumptions used.

\subsection{WiFi Model}

TABLE $\mathrm{V}$ presents the parameters used in the WiFi IEEE802.11g model. The receiver sensitivity values per modulation and coding scheme (MCS) for $\mathrm{WiFi}$ receivers are taken from [33]. The throughput efficiency, the ratio of actual IP layer throughput against physical data rate and the actual IP layer throughput of each MCS scheme are obtained from the QualNet simulator assuming fixed packet size of 1000 Bytes with constant bit rate traffic.

\begin{tabular}{cccccc}
\multicolumn{6}{c}{ TABLE V : IEEE802.11G PARAMETERS } \\
\hline Index & MCS & $\begin{array}{c}\text { Receiver } \\
\text { Sensitivity } \\
(\mathbf{d B m})\end{array}$ & $\begin{array}{c}\text { Data } \\
\text { Rate } \\
(\mathbf{M b p s})\end{array}$ & $\begin{array}{c}\text { Throughput } \\
\text { Efficiency } \\
(\boldsymbol{\varphi})\end{array}$ & $\begin{array}{c}\text { Throughput } \\
(\mathbf{M b p s})\end{array}$ \\
\hline 1 & BPSK1/2 & -94.0 & 6 & 0.70 & 4.20 \\
2 & BPSK3/4 & -93.0 & 9 & 0.64 & 5.76 \\
3 & QPSK1/2 & -91.0 & 12 & 0.61 & 7.32 \\
4 & QPSK3/4 & -90.0 & 18 & 0.54 & 9.72 \\
5 & 16-QAM1/2 & -86.0 & 24 & 0.49 & 11.76 \\
6 & 16-QAM3/4 & -83.0 & 36 & 0.41 & 14.76 \\
7 & 64-QAM2/3 & -77.0 & 48 & 0.35 & 16.80 \\
8 & 64-QAM3/4 & -74.0 & 54 & 0.32 & 17.28 \\
\hline
\end{tabular}


The path loss model in Eqn (11) is derived from field measurement detailed in [34] :

$$
P L=30.2 * \log 10(d)+22.234
$$

\subsection{LTE Model}

LTE throughput is calculated based on the throughput plot by 3GPP [35] shown in Figure 4. In the simulation, SINR ratio at a receiver is used to calculate the achievable throughput. In order to calculate the received power, the ITU recommended LTE Urban Macro (UMa) non-line-of-sight (NLOS) path loss model is used [36].

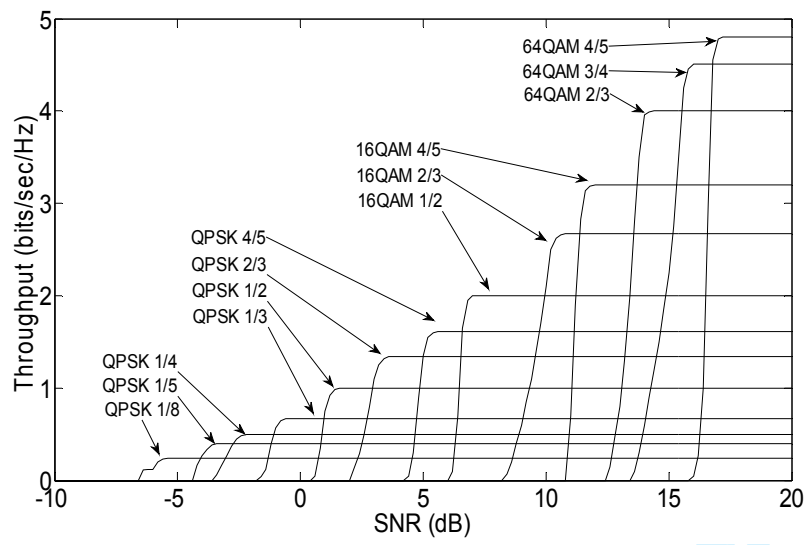

Figure 4: LTE throughput vs. SNR for different Modulation and Coding Schemes (MCS) used in the model

\subsection{Stochastic User Placement Model}

A stochastic node location model is used to position the users' in the HetNet. The authors in [37] found that this model can represent the users' locations around WiFi AP in a city or urban environment. Here users are assumed to be distributed around cluster centers. Two spatial distributions model are adopted where the first distribution is used to generate the numbers and locations of the cluster centers. The second distribution is then used for positioning the number of users around each center. The initial cluster center distribution is generated randomly with total number of cluster centers equal to $N_{\text {cluster }}$. Subsequently, a bivariate Gaussian distribution with covariance matrix $\sum=\operatorname{diag}\left(\sigma_{x}^{2}, \sigma_{y}^{2}\right)$ is used to generate actual user locations around the cluster centres. The size and shape of the cluster is characterized by the parameters $\sigma_{x}$ and $\sigma_{y}$ (Figure 5). The number of users per cluster is drawn from a Poisson distribution with intensity $N_{u s e r}$. Parameters $\sigma_{x}, \sigma_{y}$ and $N_{\text {user }}$ are the same for every cluster.

In the simulation, initially 100 users are placed in an area of 1 square kilometre with number of clusters $N_{\text {cluster }}=10$, cluster size $N_{u s e r}=10$ and $\sigma_{x}=\sigma_{y}=25$. Subsequently 50 users are added each time by increasing number of cluster $N_{\text {cluster }}$ by five while keeping the number of users per cluster $N_{\text {user }}$ and cluster dimension $\sigma$ unchanged, until the total number of users reaches 250 (Figure 6). Since all users are tabulated in an area of 1 square kilometre, the terms the number of users and user density are used interchangeably in the rest of this paper.

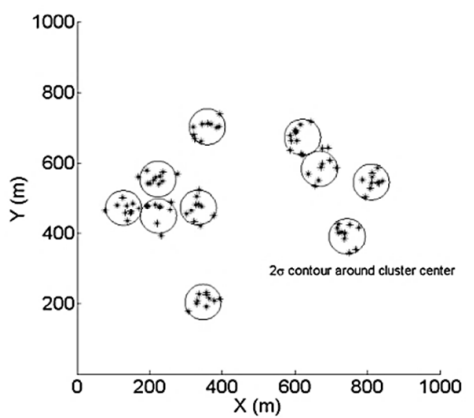

Figure 5: User placement with total number of clusters $N_{\text {cluster }}=10$, cluster size $N_{\text {user }}=10$ and $\sigma=25$

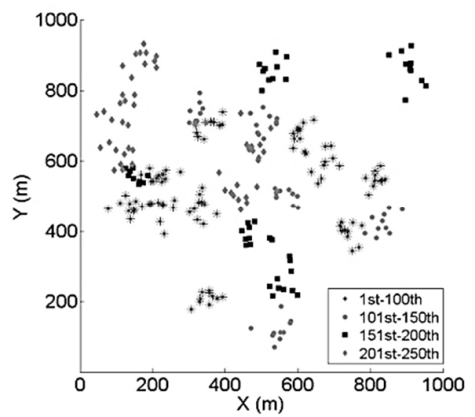

Figure 6: User placement from 100 users to 250 users with 50 users added every time, maximum $N_{\text {cluster }}=25$, cluster size $N_{\text {user }}=10$ and $\sigma=25$

\subsection{Placement of WiFi Access Points}
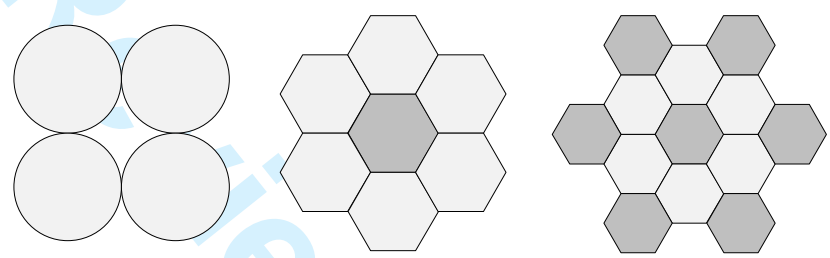

Figure 7: WiFi APs placement within HetNet that comprises the scenarios with 4, 7 and 13 WiFi APs

In the Matlab model, WiFi APs are placed within a macro LTE cell according to three selected topologies shown in Figure 7. These WiFi topologies enable the NSSs to be evaluated as a function of WiFi-LTE node ratios (or WiFi node densities). By controlling the APs' transmit power, these WiFi topologies can give the same coverage size as the macro LTE cell. For example, Figure 8a shows the coverage plot for 4 APs, while the overlay of both WiFi and LTE cell (Figure 8b) that forms the HetNet is illustrated in Figure 8c. WiFi coverage plots for 7 and 13 APs are shown in Figure 9a and Figure 9b respectively. Although there is no limit on the variation topologies which can be evaluated, the selected topologies is believed to be able to provide a good insight on the performance of NSSs under different WiFi-LTE node ratios. 


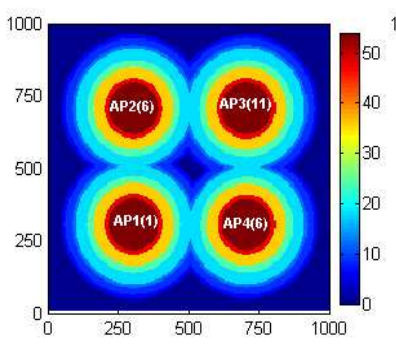

(a)

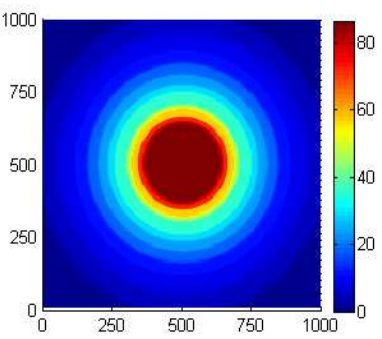

(b)

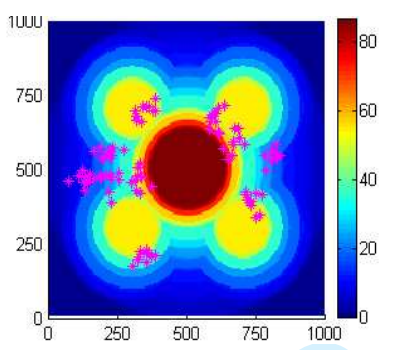

(c)

Figure 8: Network coverage plot based on data rate with $\mathrm{x}$ and $\mathrm{y}$ axis showing coverage size in meter; a) 4-WiFi-Nodes networks with access channel shown in parentheses, b) LTE network and c) HetNet (LTE+WiFi) with 100 users

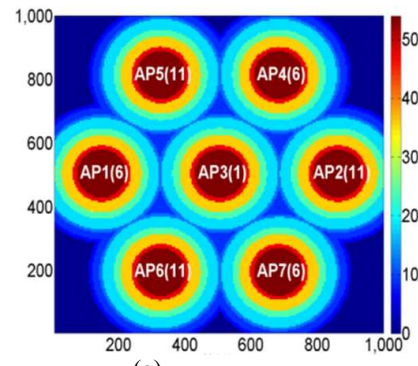

(a)



(b)
Figure 9: WiFi coverage plot based on data rate with $\mathrm{x}$ and $\mathrm{y}$ axis showing coverage size in meter; a) 7-WiFi-Nodes networks, b) 13-WiFi-Nodes networks; WiFi access channel shown in parentheses

\subsection{WiFi Access Channel and Interference}

On WiFi access channel, three non-overlapping channels with channel number 1, 6 and 11 are used (channel number is stated on top of each AP in Figure 8 and Figure 9). To calculate SINR, the simulated area is divided into 5-by-5 square meter grids. Since non-overlapping channels are used, only cochannel interference is considered. In each grid, an effective SINR is derived as:

$$
\operatorname{SINR}=\frac{p_{u}}{\sum_{t=1}^{M} p_{I}+N F_{i}}
$$

where, $p_{u}$ is the received power of user $u$ from the serving AP located in a grid, $M$ is total number of co-channel interferer APs in range, $p_{I}$ is the interference power at the receiver and $N F_{u}$ is the noise floor. Effective SINR obtained is then used to map user data rate and hence user throughput calculation in WiFi networks.



Figure 10: 7-WiFi-Nodes coverage plot with co-channel interference

Figure 10 shows the coverage plot of $7 \mathrm{WiFi}$ nodes with cochannel interference. The interference has resulted in a smaller effective WiFi coverage area as compared to Figure 9a, which has a better channel plan.

\subsection{WiFi and LTE backhaul}

In the study, WiFi backhaul capacity is varied from $1 \mathrm{Mbps}$ up to $25 \mathrm{Mbps}$ until the throughput of AP saturates (Section 4). Two backhaul scenarios are assumed when evaluating the NSSs; 1) uniform WiFi backhaul capacity and 2) non-uniform WiFi backhaul capacity throughout the HetNet. The LTE base station (BS) backhaul capacity is always assumed to be sufficient.

\subsection{General Parameters and Assumptions}

TABLE VI summarizes the simulation parameters used. Only the downlink performance is investigated in the paper. $2.6 \mathrm{GHz}$ is used for LTE as it is the frequency band allocated in Malaysia. A fixed interference margin of $3 \mathrm{~dB}$ is assumed for the LTE User Equipment (UE).

\begin{tabular}{|c|c|c|c|}
\hline & Units & WiFi 11g & LTE \\
\hline Frequency band & $\mathrm{GHz}$ & 2.4 & 2.6 \\
\hline Channel bandwidth & $\mathrm{MHz}$ & 20 & 20 \\
\hline Max EIRP & $\mathrm{dBm}$ & $27,24,18$ & 36 \\
\hline $\mathrm{Rx}$ antenna gain & $\mathrm{dBi}$ & 3 & 0 \\
\hline Antenna diversity gain & $\mathrm{dB}$ & 3 & 3 \\
\hline Noise Figure & $\mathrm{dB}$ & Not required & 10 \\
\hline Packet size & Bytes & & \\
\hline WiFi backhaul capacity & Mbps & $1-$ & \\
\hline LTE backhaul capacity & Mbps & Sufficient cap & assumed \\
\hline \multicolumn{4}{|c|}{ User Information } \\
\hline Overbooking Factor, $O F$ & - & & \\
\hline User density & Users/sqkm & 100,150 & 250 \\
\hline
\end{tabular}

Receiver antenna gain of WiFi and LTE UE is assumed to be $3 \mathrm{~dB}$ and $0 \mathrm{~dB}$ respectively. The assumption on traffic type has been described in Section 3.3.1. From [32], the Overbooking Factor (OF) is a design choice driven by actual user behavior in the deployed area. OF that varies from 4:1 to 100:1 has been reported by various ISPs and the lower the 




Figure 11: HetNet average user throughput as a function of WiFi backhaul capacity. (4 WiFi APs, 100\% WiFi-LTE overlap, Uniform WiFi backhaul capacity)

value the better the service guarantee. A factor of 10:1 in adopted to represent a relatively heavy usage scenario. User density is varied from 100 users to 250 users per square $\mathrm{km}$.

\section{Results and Analysis}

\subsection{Effect of Varying Backhaul Capacity Uniformly}

Two performance metrics namely average user throughput and user throughput fairness in the HetNet cell are used to evaluate the performance of the NSSs. Fairness is derived using Jain fairness index in the form of $\left(\sum_{u=1}^{N_{u s e r}} C_{e f f, u}\right)^{2} /$ $\left(N_{u s e r} \sum_{u=1}^{N_{u s e r}}\left(C_{e f f, u}\right)^{2}\right)[38]$.

First the performance of all NSSs is evaluated as a function of WiFi backhaul capacity from $1 \mathrm{Mbps}$ to $25 \mathrm{Mbps}$ with $100 \%$ WiFi-LTE coverage overlap and $4 \mathrm{WiFi}$ APs (as in Figure 8c). Figure 11 shows that average user throughput of PDR and DyBaCS are similar over the entire range of backhaul capacity and WF has the lowest average user throughput. The plots plateaus when backhaul capacity reaches $20 \mathrm{Mbps}$ for all NSSs as that is the maximum access throughput achievable by WiFi (IEEE $802.11 \mathrm{~g}$ ). The total $\mathrm{WiFi}$ access throughput also depends on user distribution as slower users slow down the entire network. Figure 12 evaluates the fairness in terms of achievable throughput per user. Result shows that average user throughput of PDR and DyBaCS are similar over the entire range of backhaul capacity while WF has the lowest average user throughput. DyBaCS outperforms the rest in terms of fairness and WF exhibits the worst fairness performance.

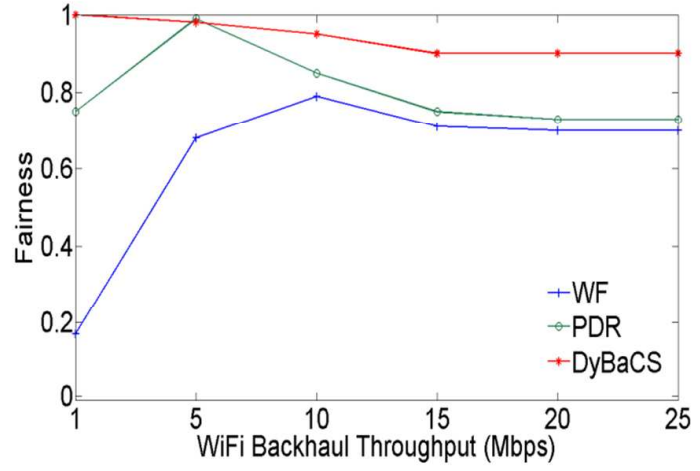

Figure 12: Fairness on bandwidth sharing as a function of WiFi backhaul capacity. (4 WiFi APs, 100\% WiFi-LTE overlap, Uniform WiFi backhaul capacity)

\subsection{Effect of WiFi-LTE Overlap Ratio Within a HetNet cell}

Since the coverage overlap between WiFi and LTE is likely to vary in practice, it is therefore important to investigate how NSSs perform in different overlap conditions. Here the coverage area is defined as a percentage e.g. $25 \%$ means $25 \%$ of LTE coverage is also covered by WiFi and $100 \%$ means all LTE coverage is overlapped by $\mathrm{WiFi}$. The simulation is carried out by switching on APs within the LTE cell one by one until the entire LTE cell overlaps with WiFi coverage. AP activation sequence is based on the number of users covers by an AP, AP which covers the most users is switched on first and followed by APs with lesser users in descending order.

Figure 13a shows that in general, that the average user throughput increases linearly for all NSSs as backhaul capacity increases and starting to flatten at $15 \mathrm{Mbps}$. The maximum average user throughput for PDR, DyBaCS and WF are $7.87 \mathrm{Mbps}, 7.60 \mathrm{Mbps}$ and $5.32 \mathrm{Mbps}$ respectively at $100 \%$ WiFi-LTE coverage overlap and 20Mbps backhaul capacity. PDR which has the highest average throughput only has a marginal performance edge as compared to DyBaCS. The difference in performances diminishes when WiFi-LTE coverage overlap increases. WF provides the worst average user throughput in most cases. An interesting point to note is for $\mathrm{WF}$ at $1 \mathrm{Mbps}$ backhaul capacity, increases in $\mathrm{WiFi}$ coverage results in a drop in the average user throughput instead of increase. This is due to the fact that WF connects all users to WiFi networks regardless of its backhaul capacity. Consequently, with limited WiFi backhaul capacity as low as $1 \mathrm{Mbps}$, WF causes the users to suffer from low average throughput and becoming more severe when WiFi coverage is larger. 


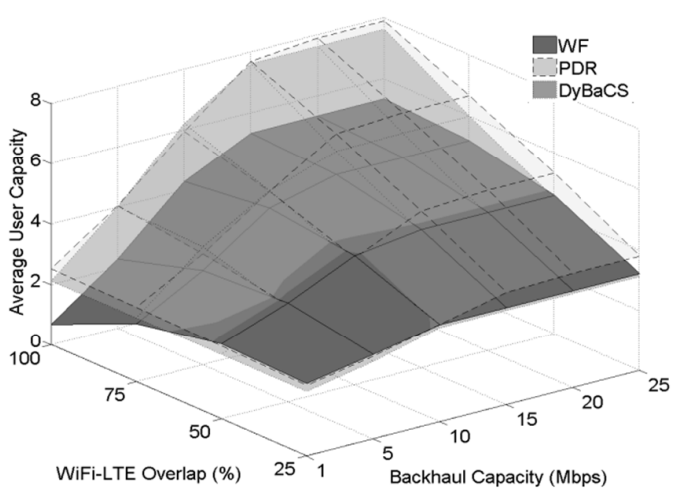

(a) Average User Throughput



(b) Fairness

Figure 13: NSSs performance as a function of backhaul capacity and WiFi-LTE overlap percentage (Maximum 4 WiFi APs, Uniform WiFi backhaul capacity).

Figure 13b shows that DyBaCS has the highest fairness for the entire range of WiFi-LTE overlap ratio and backhaul capacity considered. As for WF, higher fairness can be achieved with higher WiFi backhaul capacity because adequate capacity is shared amongst users. It is also found that fairness of PDR is closer to DyCaBS when backhaul capacity is low but the difference between them becomes larger as the backhaul capacity increases because the capacity distribution of PDR is less fair. At 25Mbps backhaul capacity and 100\% WiFi-LTE coverage overlap, both WF and PDR achieve 0.7 and 0.73 respectively in terms of fairness, but this is lower than 0.9 by DyBaCS.

\subsection{Effect of User Density}

A high probability that the number of users in a network will vary drastically from peak hour to off peak of the day exists, especially in urban area. Furthermore, the number of users may also increase but gradually in residential areas due to the growth in population. To evaluate how the NSSs under study cope with the variation of user density, an evaluation is carried out by varying the number of users in the HetNet cell. A scenario with $7 \mathrm{WiFi}$ APs, a medium WiFi density, is chosen and WiFi-LTE Coverage overlap is set to $100 \%$. The number of users is increased from 100 to 250 , in 50 user increments (Figure 14).

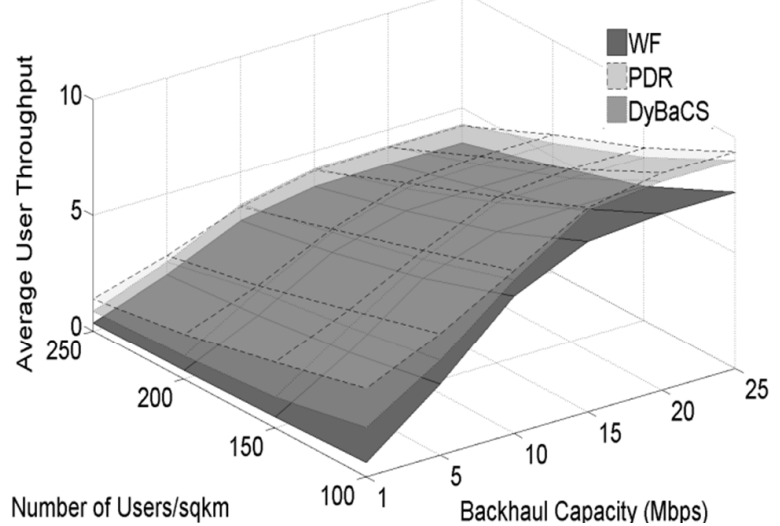

(a) Average User Throughput

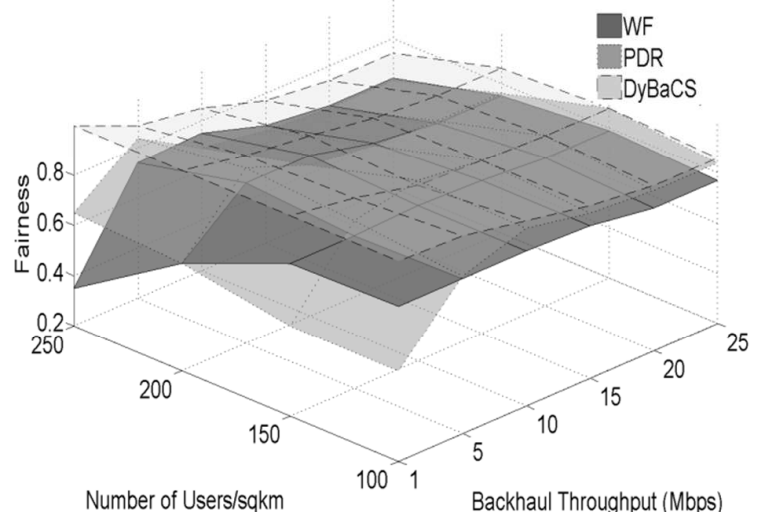

(b) Fairness

Figure 14: NSSs performance as a function of Number of Users and Backhaul capacity. (7 WiFi APs, 100\% WiFi-LTE Coverage)

Figure 14 shows that PDR provides an advantage on average user throughput over DyBaCS when WiFi backhaul capacity values are low (i.e. $1 \mathrm{Mbps}$ to $5 \mathrm{Mbps}$ ) regardless of user density. However, when the WiFi backhaul capacity is increased, the difference in average user throughput between PDR and DyBaCS becomes negligible. At highest user density with 250 users per square kilometer, average user throughput of DyBaCS is greater than PDR when WiFi backhaul capacity exceeds $10 \mathrm{Mbps}$. WF remains the worst in providing capacity to users. DyBaCS remains the best scheme in terms of fairness for the entire range of user density and backhaul capacity.

Figure 14 implies that with increasing number of users, PDR is actually losing its advantage on average user throughput and fairness to DyBaCS and WF respectively, accentuated in Figure 15 which depicts NSSs performance for all three WiFi node densities considering the highest number of users (250 users). Figure 15 shows that with 250 users, PDR is the worst in terms of fairness despite previous results (refer to Figure 13 and Figure 14) showing that PDR is the second best on fairness. PDR's advantage on average user throughput is also sliced to a thin margin when both number of users and WiFi node density is high. For instance, in Figure 15, when number of WiFi nodes is 4, PDR is much superior in terms of average user throughput. 


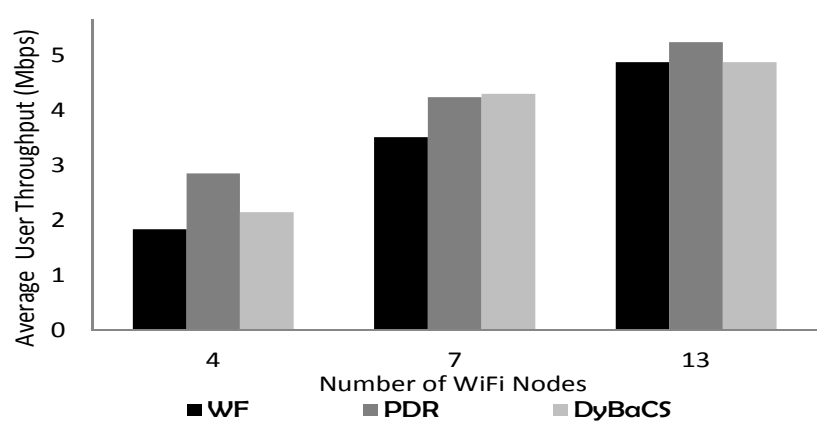

(a) Average User Throughput

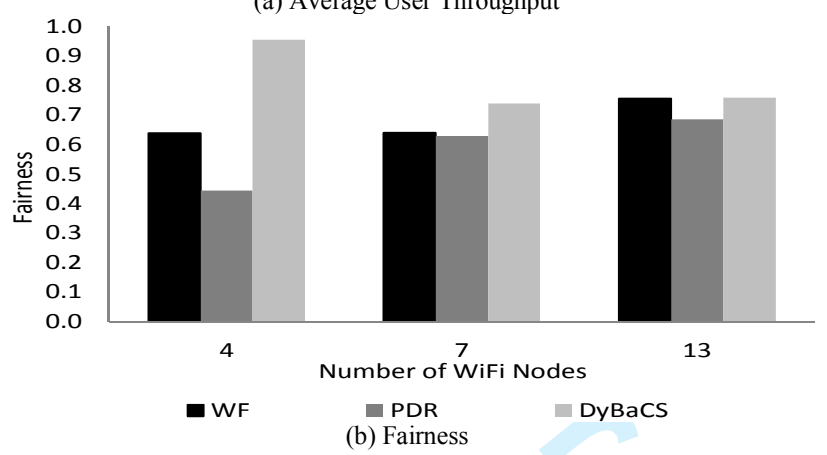

Figure 15: Performance comparison between NSSs as a function of total WiFi Nodes with 250 Users and fixed backhaul capacity of $20 \mathrm{Mbps}$

However, at higher WiFi node density, PDR's advantage on average user throughput becomes insignificant.

As a general conclusion DyBaCS is good for fairness whilst maintaining the average user throughput, a clear advantage when the number of users is high with dense AP deployment, a typical urban scenario. It is adaptive to varying number of WiFi nodes and varying number of users, highly relevant in fast changing environments. PDR which is a form of greedy algorithm maximizes the average user throughput but at the expense of compromised fairness and its advantages in cell throughput diminish when both number of users and AP density are high. Although WF performs adequately compared to PDR when APs density, user density and backhaul capacity are high, it performs poorly otherwise and never outperforms DyBaCS in all scenarios under study.

\subsection{Performance on Non-uniform Backhaul Capacity}

In another scenario with 100 users, backhaul capacity of 4 APs covering the entire HetNet are non-uniformly assigned with AP1, AP2, AP3 and AP2 equal to $10 \mathrm{Mbps}, 0.5 \mathrm{Mbps}$, $20 \mathrm{Mbps}$ and $3 \mathrm{Mbps}$ respectively. Average user throughput is presented in Figure 16a, where PDR has the highest value (4.75Mbps) followed by DyBaCS (4.22Mbps) and WF (2.62Mbps). PDR is expected to offer higher average user throughput as it selects the network based on the highest physical data rate with the intention of maximizing the average user throughput. However, it degrades the fairness in terms of capacity distribution among the users highlighted in Figure 16b where the fairness index of PDR is only 0.59 compared to 0.86 by using DyBaCS. Although the average throughput of DyBaCS is $11 \%$ lower than PDR, but the traded off seems to be worthwhile as fairness is significantly improved by $45 \%$. WF only provides a 0.43 fairness index.
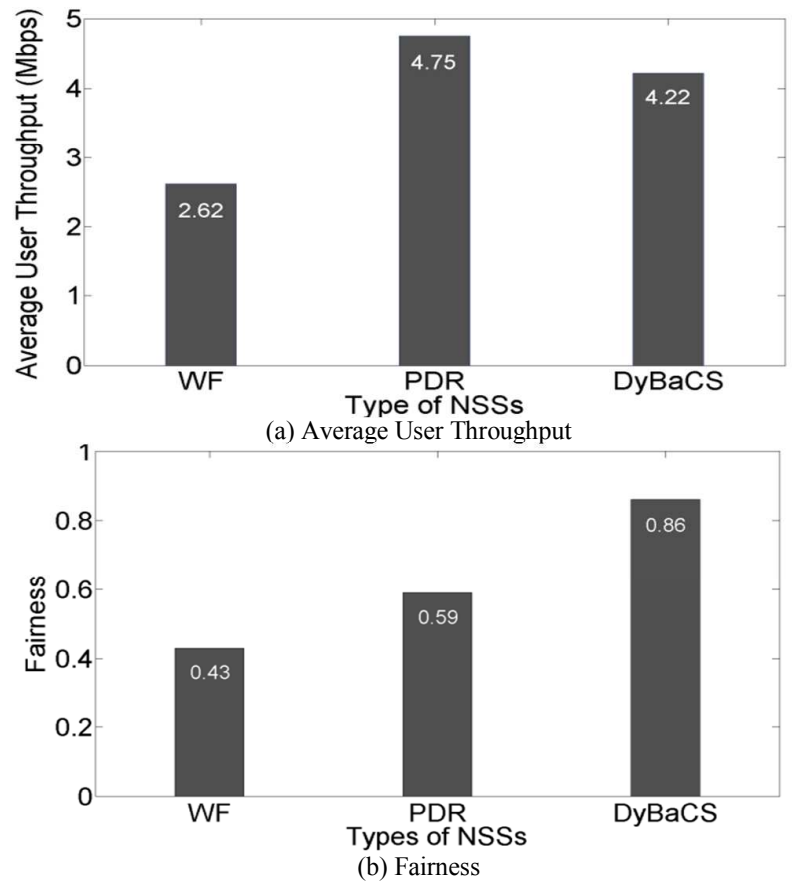

Figure 16: (a) Average user throughput and (b) Fairness on bandwidth sharing with non-uniform WiFi backhaul capacity. (4 Nodes, 100 users)

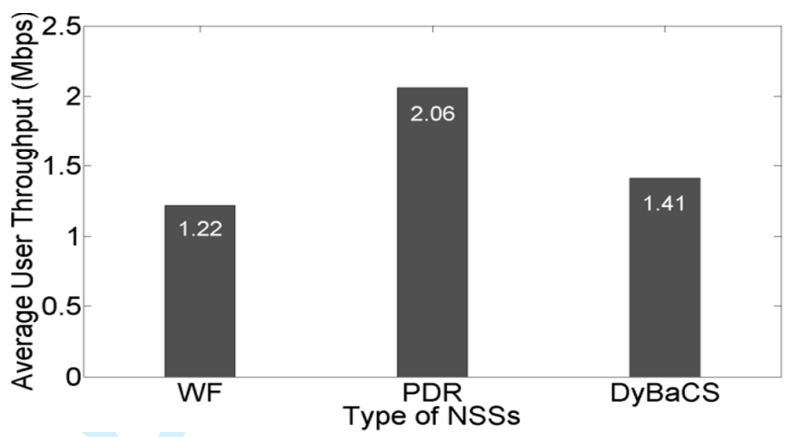

(a) Average User Throughput



(b) Fairness

Figure 17: (a) Average user throughput and (b) Fairness of bandwidth sharing with non-uniform WiFi backhaul capacity. (4 Nodes, 250 users)

Using a similar scenario but by increasing the number of users to 250 , Figure $17 \mathrm{~b}$ shows that fairness index offered by PDR (at 0.33) is the poorest amongst all the NSSs under study although as shown in Figure 
$17 \mathrm{a}$, PDR provides the highest average user throughput. DyBaCS on the other hand provides a good balance between average user throughput and fairness.

The results captured in Figure 16 and Figure 17 consistently indicate that $\mathrm{DyBaCS}$, under non-uniform WiFi backhaul scenarios, provisions best fairness to users with minimal penalty in the average user throughput. DyBaCS provides the best performance on both average throughput and fairness when both user density and WiFi node density is high (similar to uniform backhaul capacity case). Performance under non-uniform $\mathrm{WiFi}$ backhaul is in agreement with that of uniform backhaul evaluation detailed in Section 4. However, it is important to note that changes in user location and distribution of backhaul capacity may affect performance to a certain degree but the general trends remain.

\section{Conclusions}

A backhaul capacity sensitive network selection scheme referred to as DyBaCS within a wireless HetNet has been presented. The performance of DyBaCS and two commonly used NSSs has been evaluated as a function of WiFi backhaul capacity, WiFi-LTE coverage ratio, $\mathrm{WiFi}$-LTE node ratio and user density. The performance of NSSs has also been compared under uniform and non-uniform WiFi backhaul capacity distributions. Results show that the proposed DyBaCS scheme provides the best fairness whilst preserving the average user throughput over all scenarios. DyBaCS is a highly scalable NSS as compared to PDR and WF in scenarios with varying number of users, WiFi nodes and WiFi backhaul capacity. Future work needs to consider more realistic proportional fairness capacity sharing schemes. The use of higher capacity LTE base stations and IEEE802.11n WiFi also needs to be studied. Besides, evaluation under different traffic types also needs to be addressed.

\section{Acknowledgment}

The work is supported by Ministry of Science, Technology and Innovation of Malaysia under the Science Fund project code 01-03-04-SF0011.

\section{References}

1. A Ting, D Chieng, K H Kwong, I Andonovic \& K D Wong. Dynamic backhaul sensitive Network Selection Scheme in LTE-WiFi wireless HetNet. IEEE 24th International Symposium on Personal Indoor and Mobile Radio Communications (PIMRC); 8-11 Sept 2013: 30613065 .

2. The LTE capacity shortfall: Why small cell backhaul is the answer, Aviat Networks, Retrieved 14th May 2013 from http:// www.aviatnetwork.com/

3. Small cell market status Feb 2013, Informa telecoms \& media, Retrieved 2nd October 2013 from http://www.smallcellforum.org/

4. Small cell operators face myriad operational and financial challenges, Infonetics Research, Retrieved 1st Oct 2013 from http://www.infonetics.com/

5. Mark Priscaro (14th May 2013), Telecom New Zealand and Ruckus Wireless Transform Traditional Phone
Booths into Super Fast Wi-Fi Hotspots, Ruckus Wireless, Retrieved 16th May 2013 from http:// www.ruckuswireless.com/

6. Firetide Wireless Mesh Solutions for TELCOS: Access \& Small Cell Backhaul, https://www.winncom.com/manufacturer/firetide

7. Karam F W, Jensen T. Performance analysis of ranking for QoS handover algorithm for selection of access network in heterogeneous wireless networks. 21st International Conference onComputer Communications and Networks (ICCCN), 30 July-2 Aug 2012:1-6.

8. A Handa. Mobile Data Offload for $3 \mathrm{G}$ Networks: A White Paper. http:/www.intellinettech.com/Media/PagePDF/Data\%20Offload.pdf, Oct. 2009.

9. J Nie, J Wen, Q Dong, Z Zhou. A seamless handoff in IEEE802.16a and IEEE802.11n hybrid networks, in proc. Int. Conf. Communi. Circuits Syst 2005, 1: 383-387.

10. J Roy, V Vaidehi, and S Srikanth. Always best-connected QoS integration model for the WLAN, WiMAX heterogeneous network. In IEEE First International Conference on Industrial and Information Systems, 8-11 Aug 2006: 361-366.

11. Y Bejerano, S J Han, and L E Li. Fairness and Load Balancing in Wireless LANs Using Association Control, IEEE/ACM Transactions on Networking 2007; 15(3):560573.

12. Judd G, Steenkiste P. Fixing 802. 11 access point selection. ACM SIGCOMM Computer Communication Review 2002; 32(3): 31-31.

13. D Kumar, E Altman, and J M Kelif. Globally optimal user-network association in an 802.11 WLAN and 3G UMTS hybrid cell. International Teletraffic Congress, Ottawa, Canada, June 17-21 2007: 1173-1187.

14. K Premkumar and A Kumar. Optimum Association of Mobile Wireless Devices with a WLAN-3G Access Network, Proc. IEEE Int'l Conf. Comm. (ICC '06), 11-15 June 2006: 2002-2008.

15. S. Singh, H. S. Dhillon, and J. G. Andrews. Offloading in heterogeneous networks: Modeling, analysis, and design insights, IEEE Trans. Wireless Communications 2013; 12(5): 2482-2497.

16. P Li , J Kong, S H Kim, H B Jung, X L Niu, D K Kim. Power allocation with max-min fairness for multicast in heterogeneous network, 14th International Conference on Advanced Communication Technology (ICACT), 19-22 Feb 2012: 502-505

17. P Xue, P Gong, J H Park, D Y Park, D K Kim. Radio resource management with proportional rate constraint in the heterogeneous networks, IEEE Transactions on Wireless Communications 2011; 11(3): 1066-1075.

18. Y Bejerano and S J Han. Cell Breathing Techniques for Load Balancing in Wireless LANs. IEEE Tranctions on Mobile Computing 2009; 8(6): 735-749.

19. H Velayos, V Aleo, and G Karlsson. Load Balancing in Overlapping Wireless LAN Cells, Proc. IEEE International Conference on Communications, 20-24 June 2004: 3833-3836.

20. Pei X, Jiang T, Qu D, Zhu G \& Liu J. Radio-resource management and access-control mechanism based on a novel economic model in heterogeneous wireless 
networks. IEEE Transactions on Vehicular Technology 2010, 59(6): 3047-3056.

21. Zhuo X, Hua S, Gao W \& Cao G. An Incentive Framework for Cellular Traffic Offloading. IEEE Transactions on Mobile Computing 2014; 13(3): 541-555.

22. Yang $\mathrm{Z}$, Yang Q, Fu F \& Kwak K S. A novel load balancing scheme in LTE and WiFi coexisted network for OFDMA system. International Conference on Wireless Communications \& Signal Processing (WCSP), 24-26 Oct 2013; $1-5$.

23. F Bari and V C M Leung. Automated network selection in a heterogeneous wireless network environment. IEEE Network 2007; 21(1): 34-40.

24. H Hu, W Zhou, S Zhang, J Song. A Novel network selection algorithm in next generation heterogeneous network for modern service industry. IEEE Asia-Pacific Services Computing Conference, 9-12 Dec 2008: 12631268.

25. Galeana-Zapien H, Ferrus R. Design and Evaluation of a Backhaul-Aware Base Station Assignment Algorithm for OFDMA-Based Cellular Networks. IEEE Transactions on Wireless Communications 2010; 9(10): 3226-3237.


Networks, $2^{\text {nd }}$ Edition, John Wiley \& Sons, 2005.

27. P Bender et al. CDMA/HDR: A Bandwidth Efficient High-speed Wireless Data Service for Nomadic User. IEEE Communication Magazine 2000; 38(7): 70-77.

28. Gerasimenko M, Moltchanov D, Florea R, Andreev S, Koucheryavy Y, Himayat N, Yeh S-p. \& Talwar S. Cooperative Radio Resource Management in Heterogeneous Cloud Radio Access Networks. IEEE Access 2015; 3: 397-406.

29. Yang $\mathrm{C}, \mathrm{Li}$ J \& Anpalagan A. Cooperative bargaining game - theoretic methodology for $5 \mathrm{G}$ wireless heterogeneous networks. Transactions on Emerging Telecommunications Technologies 2014; 26(1): 70-81.
30. Bredel M \& Fidler M. A measurement study of bandwidth estimation in IEEE $802.11 \mathrm{~g}$ wireless LANs using the DCF. NETWORKING 2008 Ad Hoc and Sensor Networks, Wireless Networks, Next Generation Internet; 4928: 314325. Springer.

31. Yongmin Choi, Hyun Wook Ji, Jae-yoon Park, Hyun-chul Kim, Silvester J A. A $3 \mathrm{~W}$ network strategy for mobile data traffic offloading. IEEE Communications Magazine 2011; 49(10): 118-123.

32. Tranzeo Wireless Technologies Inc. Example Community Broadband Wireless Mesh Network Design, Version 1.1, 20June 2007.

33. NanoStation2, Ubiquiti Networks, www.ubnt.com/products/nano.php

34. A Ting, D Chieng, $\mathrm{K} \mathrm{H}$ Kwong, I Andonovic. Optimization of Heterogeneous Multi-radio Multi-hop Rural Wireless Network. IEEE $14^{\text {th }}$ International Conference on Communicaiton Technology 2012, ChengDu China, 9-11 Nov 2012: 1159-1165.

35. 3GPP, Evolved Universal Terrestrial Radio Access (EUTRA); Radio Frequency (RF) system scenarios (Release 10), 3GPP TR 36.942 V10.2.0 2010

36. ITU-R Report M.2135-1: Guidelines for evaluation of radio interface technologies for IMT-Advanced, December 2009.

37. M Petrova, P Mähönen, and J Riihijärvi. Connectivity analysis of clustered ad hoc and mesh networks, In Proceedings of IEEE GLOBECOM, Martinique 26-30 Nov 2007: 1139-1143.

38. R Jain, A Durresi, and G Babic. Throughput fairness index: an explanation, ATM Forum/99-0045, Feb. 1999. 